PNL-2930

UC-23

\title{
LIST OF DOE RADIOISOTOPE CUSTOMERS \\ WITH SUMMARY OF RADIOISOTOPE SHIPMENTS \\ FY 1978
}

Compiled by

J. S. Burlison

R. I. Laidler

May 1979

\author{
Prepared for \\ Division of Financial Services \\ Office of the Assistant Secretary for Environment \\ U.S. Department of Energy \\ under Contract No. EY-76-C-06-1830
}

Pacif ic Northwest Laboratory

Richland, Washington 99352

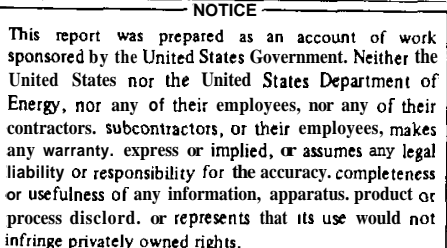

PUBLICLYRELEASABLE arez E Ditham Authơfizing Official

Date: $06 / 13 / 2007$ 


\section{DISCLAIMER}

This report was prepared as an account of work sponsored by an agency of the United States Government. Neither the United States Government nor any agency Thereof, nor any of their employees, makes any warranty, express or implied, or assumes any legal liability or responsibility for the accuracy, completeness, or usefulness of any information, apparatus, product, or process disclosed, or represents that its use would not infringe privately owned rights. Reference herein to any specific commercial product, process, or service by trade name, trademark, manufacturer, or otherwise does not necessarily constitute or imply its endorsement, recommendation, or favoring by the United States Government or any agency thereof. The views and opinions of authors expressed herein do not necessarily state or reflect those of the United States Government or any agency thereof. 


\section{DISCLAIMER}

Portions of this document may be illegible in electronic image products. Images are produced from the best available original document. 


\section{ACKNOWLEDGMENTS}

The author wishes to thank the following laboratories and DOE Area Offices for their annual contributions from which this report is compiled.

Argonne National Laboratory

Brookhaven National Laboratory

Hanford Engineering Development Laboratory

Idaho Operations Office/Aerojet Nuclear Company

Los Alamos Scientific Laboratory

Mound Fac i 1 ity

Pacific Northwest Laboratory

Savannah River Plant/DOE

UNC Nuclear Industries, Inc.

\section{PREVIOUS REPORTS}

Previous reports in this series are:

TID-2160 (FY 1964)

ORNL-IIC-6 (FY 1965)

ORNL-IIC-9 (FY 1966)

ORNL-IIC-13 (FY 1967)
ORNL-IIC-17 (FY 1968)

ORNL-IIC-27 (FY 1969)

ORNL-IIC-32 (FY 1970)

ORNL-IIC-38 (FY 1971) $\begin{array}{lll}\text { BNWL-1774 } & \text { (FY 1972) } \\ \text { BNWL-1824 (FY 1973) } \\ \text { BNWL-1902 } & \text { (FY 1974) } \\ \text { BNWL-1993 } & \text { (FY 1975) } \\ \text { BNWL-2147 } & \text { (FY 1976) } \\ \text { PNL-2572 } & \text { (FY 1977) }\end{array}$

Similar reports covering stable isotope distribution have also been prepared and are available from the National Technical Information Center, U.S. Department of Commerce, 5285 Port Royal Rd, Springfield, VA 22161.

- "Stable Isotope Customer List and Summary of Shipments, FY 1977," Oak Ridge National Laboratory, ORNL/TM-6241, Apri1 1978 (\$4.50 printed; \$3.00 microfiche).

- "Stable Isotope Sales: Mound Facility Customer and Shipment Summaries, FY 1978." MLM-2594, March 16, 1979 (\$4.00 printed; $\$ 3.00$ microfiche).

- "Stable Isotope Sales: Mound Facility Customer and Shipment Summaries, FY 1977." MLM-2504, April 27, 1978 (\$4.00 printed; $\$ 3.00$ microfiche).

- "Stable Isotope Sales: Mound Facility Customer and Shipment Summaries, FY 1976 and 1976 A." MLM-2403, June 6, 1977 (\$4.00 printed; \$3.00 microfiche).

- "Stable Isotope Sales: Mound Facility Customer and Shipment Summaries, FY 1975." MLM-2295, April 30, 1976 ( $\$ 4.00$ printed; $\$ 3.00$ microfiche). 
AKNOWLEDGMENTS . . . . . . . . . . . . . . . . . . . . . . . . . . . . .

PREVIOUSREPORTS. . . . . . . . . . . . . . . . . . . . . . . . . .

NOMENCLATURE . . . . . . . . . . . . . . . . . . . . . . . . . . . v v

INTRODUCTION . . . . . . . . . . . . . . . . . . . . . . . . . . . 1

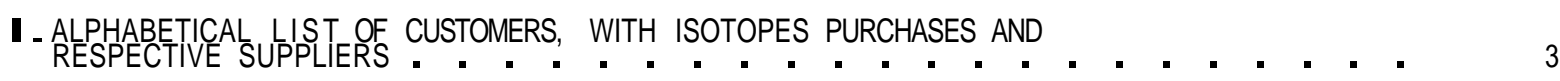

A. DOMESTIC . . . . . . . . . . . . . . . . . . . . . . . . . . . . 3

B. FOREIGN. . . . . . . . . . . . . . . . . . . . . . . . 22

11. ALPHABETICAL LIST OF ISOTOPES CROSS-REFERENCED TO CUSTOMER NUMBERS. . . . . . . . 29

A. DOMESTIC. . . . . . . . . . . . . . . . . . . . . . . . . 29

B. FOREIGN. . . . . . . . . . . . . . . . . . . . . . . 33

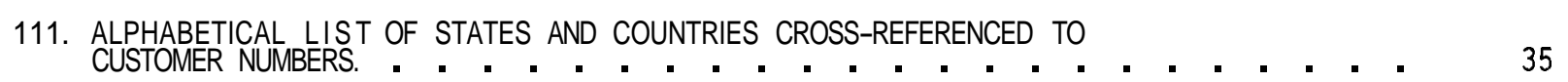

A. DOMESTIC. . . . . . . . . . . . . . . . . . . . . . . . . 35

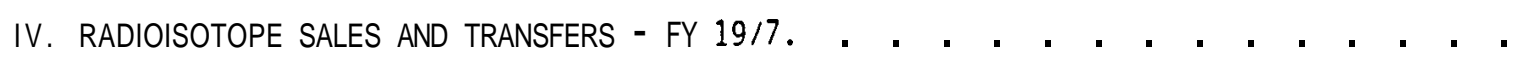



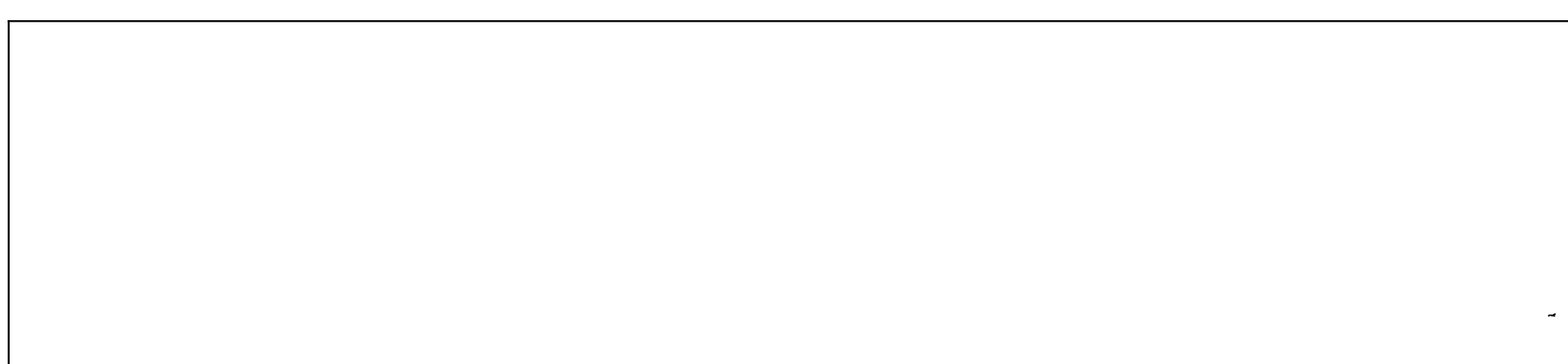

. 


\section{NOMENCLATURE}

I CLASSIFICATIONS

Cyclotron Service

Irradiations

Depleted Uranium

Encapsulation

Enriched Uranium

Fission Products

Miscellaneous Compound

Preparations

11. ISOTOPE SUPPLIER AND CONTACT

Argonne National Laboratory

J. C. Haugen

Special Materials Division

Argonne National Laboratory

9700 S. Cass Ave

Argonne, IL 60439

FTS 972-6778

Commercial 312-972-6778

Battelle, Pacific Northwest Laboratories(a)

(P)

J. V. Filsinger

Safety and Nuclear Materials Management

Pacific Northwest Laboratory

Richland, WA 99352

FTS 444-2051

Commercia 1 509-946-2051

Brookhaven National Laboratory

P. Colsman

Isotopes and Special Materials Group

Brookhaven National Laboratory

FTS 664-4058

Commercial 516-345-3441

Extension 4058

CSI

Cap

FP

MCP

(A)

(B)
Natural Uranium

$\mathrm{NU}$

Reactor Service Irriadiations

R S I

DU

Withdrawn radioisotopes prepared under special approval

RW

EU

Special Services

SS

Targets

Tgs

Hanford Engineering Development Laboratory

(H)

D. Engel, Manager

Materia1s Management

Richland, WA 99352

FTS 444-3548

Commercial 509-942-3548

Idaho Operations Office

R. E. Simonds, Director

Idaho National Engineering Co.

EG\&G Idaho, Inc.

Po Box 1625

Idaho Falls, ID 83401

FTS 583-4266

Commercial 208-526-4266

Extension 4266

Los Alamos Scientific Laboratory

(I)

C. Longmire

Los Alamos Scientific Laboratory

University of California

PO Box 1663

Los Alamos, NM 87545

FTS 843-4948

Commercia 1 505-667-4948

(a) Battelle Memorial Institute operates Pacific Northwest Laboratory for the Department of Energy. In this report, we have identified Pacific Northwest Laboratory as Battelle, Pacific Northwest Laboratories. 
NOMENCLATURE (contd. )

Mound Facility

R. DeWitt

Sa 1es/Mark e ting Manager

Stable Isotope Sales

Monsanto Research Corporation

P.0. Box 32

Miamisburg, $\mathrm{OH} \quad 45342$

FTS $774-3896$

Commerc ia) 513-865-3896

Extension 7147

Oak Ridge National Laboratory

J. E. Ratledge, Supervisor Isotope Sales

Oak Ridge National Laboratory

P.0. BOX $X$

Oak Ridge, TN 37830

FTS 624-6984

Commercia 1 615-574-6984

Extension 6661
(M)

$$
\text { Savannah River Plant }
$$

M. J. Sires, Acting Director

Technical and Production Division

Department of Energy

P.0. Box A

Aiken, SC 29801

FTS 239-2217

Commerc ia $1803-725-6211$

Extension 2217

UNC Nuclear Industries, Inc.

(U)

P. A. Carlson, Manager

Reactor Technology

Richland, WA 99352

FTS 444-1631

Commercial 509-942-1631 
LIST OF DOE RADIOISOTOPE CUSTOMERS

WITH SUMMARY OF RADIOISOTOPE SHIPMENTS

FY 1978

INTRODUCTION

The fourteenth edition of the radioisotope customer list was prepared at the request of the Division of Financial Services, Office of the Assistant Secretary for Environment, Department of Energy (DOE). The purpose of the document is to list DOE's radioisotopes production and distribution activities by its facilities at Argonne National Laboratory; Pacific Northwest Laboratory; Brookhaven National Laboratory; Hanford Engineering Development Laboratory; Idaho Operations Office; Los Alamos Scientific Laboratory; Mound Facility; Oak Ridge National Laboratory; Savannah River Laboratory; and UNC Nuclear Industries, Inc.

The information is divided into four sections. Section I is an alphabetical list of domestic and foreign customers and their addresses. Each customer has been assigned a number according to its alphabetical position to provide a means of cross-referencing in the following sections. The isotopes purchased are listed after the address of the customer, and the laboratory supplying each isotope is indicated by a letter set off by parentheses (see page vi, Nomenclature, Part II), (a) Section II is an alphabetical list of isotopes that are cross-referenced to customer numbers and divided into the domestic and foreign catagories. This section provides a quick idea 'of the number of companies purchasing a particular isotope. If more information is needed, the reader can locate the customer by number and determine the laboratory supplying the isotope. Section III is an alphabetical list of states and is also cross-referenced to customer numbers, indicating geographical concentrations of isotope users. Section IV summarizes the FY-1978 radioisotope shipment activities of laboratories in a comprehensive table providing an alphabetical listing of the isotopes and their suppliers. The shipments, quantities, and dollars are broken down for each isotope under the domestic, foreign, and project (DOE facilities) categories, and the total figures for each isotope are also provided.

(a) All entries for Argonne, UNC, and Idaho are either reactor service irradiations, cyclotrons service irradiations, or both with no processed isotopes being sold; entries for Savannah River represent material for resale and loans. 
I - ALPHABETICAL LIST OF CUSTOMERS, WITH CONTACTS AND ISOTOPES PURCHASED

\section{A. DOMESTIC}

1 Aerojet Nuclear Company

550 Second Street

P.O. Box 1845

Idaho Falls, Idaho 83401

${ }^{252} \mathrm{Cf}(0),{ }^{85} \mathrm{Kr}(0),{ }^{236} \mathrm{Pu}(0), \mathrm{SS}(0)$, $\operatorname{Tgs}(0),{ }^{133} \mathrm{Xe}(0),{ }^{95} \mathrm{Zr}(0)$

2 Aerojet Ordnance Manufacturing Co.

3097 E. Ana Street

Compton, California 90221

$D U(P)$

A ir Force Academy,

see United States A ir Force Academy

3 Airco, Inc.

Central Research Laboratories

Murray Hill, New Jersey 07974

$85_{\mathrm{Kr}}(0),{ }^{133} \mathrm{Xe}(0)$

Alabama, University of, see University of Alabama

4 Albert Einstein College of Medicine Ullman Building, Room 121

1300 Morris Park Avenue

Bronx, New York 10461

$81_{\mathrm{Kr}}(\mathrm{B})$

$5 \quad$ Allen-Bradley Company

136 W. Greenfield Avenue

Milwaukee, Wisconsin 53204

$85_{\mathrm{Kr}}(0)$

6 Allied Chemical Corporation

Idaho Chemical Programs - Operations

Office

550 2nd Street

Idaho Falls, Idaho 83401

${ }^{198} \mathrm{Au}$ ( I ),${ }^{60} \mathrm{Co}(\mathrm{I})$

\footnotetext{
*All starred isotopes are Oak Ridge billed.
}

7 American Atomics Corporation

425 S. Plumer Avenue

Tuscon, Arizona 85719

${ }^{3} H(0),{ }^{85} \mathrm{Kr}(0),{ }^{238} \mathrm{U}(\mathrm{M})$

8 Amersham Corporation

2636 S. Clearbrook Drive

Arlington Heights, Illinois 60005

${ }^{241} \mathrm{Am}(0), \operatorname{Cap}(0),{ }^{109} \mathrm{Cd}(\mathrm{B}), * \operatorname{CSI}(0)$, ${ }^{3} \mathrm{H}(0),{ }^{129} \mathrm{I}(0),{ }^{106} \mathrm{Ru}(0), \operatorname{Tgs}(0)$

9 AMOCO 0 i1 Company

Research and Development

P.O. Box 400

Naperville, I11 ino is 60540

$\operatorname{RSI}(A)$

10 ARCO Nuclear Company

P.0. Box 546

Leechburg, Pennsylvania 15656

${ }^{238} \mathrm{Pu}(0)$

11 Argonne National Laboratory

9700 South Cass Avenue

Argonne, Illinois 60439

${ }^{249} \mathrm{Bk}(0),{ }^{252} \mathrm{Cf}(\mathrm{S}),{ }^{248} \mathrm{Cm}(0), \mathrm{DU}(\mathrm{P})$, $253_{\mathrm{Es}(0)}{ }^{257} \mathrm{Fm}(0),{ }^{95} \mathrm{Nb}(0), \mathrm{Np}(P)$, ${ }^{237} \mathrm{~Np}(0),{ }^{236} \mathrm{Pu}(0),{ }^{242} \mathrm{Pu}(0),{ }^{106} \mathrm{Ru}(0)$, ${ }^{99} \mathrm{Tc}(0), \mathrm{Tgs}(0),{ }^{230} \mathrm{Th}(0){ }^{232} \mathrm{Th}(0)$, ${ }^{233} U(0),{ }^{235} U(0),{ }^{238} U(0),{ }^{91} Y(0)$, $95 \operatorname{Zr}(0)$

12 Argonne National Laboratory P.O. Box 2528

Idaho Falls, Idaho 83401

${ }^{128}$ xe (stable) (I)

13 Argonne National Laboratory

Scovi1le, Idaho 83401

${ }^{58} \mathrm{Co}(\mathrm{H}),{ }^{55} \mathrm{Fe}(\mathrm{H}),{ }^{54} \mathrm{Mn}(\mathrm{H}),{ }^{56} \mathrm{Mn}(\mathrm{H})$, ${ }^{182} \mathrm{Ta}(\mathrm{H})$ 
14 Arizona State University

Tempe, Arizona 85281

${ }^{252} \mathrm{Cf}(0), \operatorname{Tgs}(0)$

Arizona, University of, see University of Arizona

Arkansas, University of, see University of Arkansas

Amy, see United States Amy

15 Atomic International

8900 DeSota Avenue

Canoga Park, California 91304

${ }^{58} \mathrm{Co}(\mathrm{H}),{ }^{56} \mathrm{Mn}(\mathrm{H}),{ }^{182} \mathrm{Ta}(\mathrm{H})$

16 Automation Industries, Inc.

P.0. Box 245

Phoenixville, Pennsylvania 19460

${ }^{192} \operatorname{Ir}(0)$, RW(0)

17 Babcock and Wilcox Company

Research and Development Division

1562 Beeson Street

Alliance, Ohio 44601

${ }^{237} \mathrm{~Np}(0),{ }^{244} \mathrm{Pu}(0), \operatorname{Tgs}(0),{ }^{233} \mathrm{U}(0)$, $238 \mathrm{U}(0)$

Baker Chemical Company, J. T., see

J. T. Baker Chemical Company

18 Battelle Memorial Institute

505 King Avenue

Columbus, Ohio 43201

${ }^{252} \mathrm{Cf}(0), \mathrm{NU}(\mathrm{P}),{ }^{236} \mathrm{Pu}(0), \mathrm{SS}(0)$,

232 U(0)
19 Battelle, Pacific Northwest Laboratories, P.O. Box 999

Richland, Washington 99352

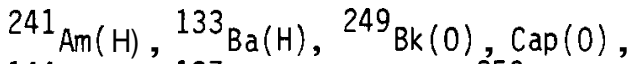
${ }^{144} \mathrm{Ce}(\mathrm{O}),{ }^{137} \mathrm{Cs}(\mathrm{H}), \mathrm{CSI}(0),{ }^{253} \mathrm{Es}(0)$, $152 \mathrm{Eu}(\mathrm{H}),{ }^{3} \mathrm{H}(0),{ }^{85} \mathrm{Kr}(0),{ }^{95} \mathrm{Nb}(0)$, ${ }^{231} \mathrm{~Pa}(\mathrm{O}),{ }^{147} \mathrm{Pm}(\mathrm{H}),{ }^{238} \mathrm{Pu}(\mathrm{H}),{ }^{239} \mathrm{Pu}(\mathrm{H})$, ${ }^{236} \mathrm{Pu}(0),{ }^{244} \mathrm{Pu}(0), \operatorname{RaDEF}(\mathrm{H}), \mathrm{SS}(0)$, ${ }^{90} \operatorname{SrY}(H),{ }^{99} \mathrm{Tc}(0), \operatorname{Tgs}(0),{ }^{170} \mathrm{Tm}(U)$, $\mathrm{U}$ (natura 1$)(\mathrm{H}),{ }^{95} \operatorname{Zr}(0)$

20 Bell Telephone Laboratories Murray Hill, New Jersey 07971

$M C P(0), S S(0)$

21 Brookhaven National Laboratory Upton, Long Island, New York 11973 ${ }^{241} \mathrm{Am}(0),{ }^{252} \mathrm{Cf}(0),{ }^{252} \mathrm{Cf}(\mathrm{S}),{ }^{3} \mathrm{H}(0)$, ${ }^{238} \mathrm{Pu}(0),{ }^{244} \mathrm{Pu}(0),{ }^{103} \mathrm{Ru}(0), \operatorname{Tgs}(0)$, ${ }^{232} \operatorname{Th}(0)$

22 Brown University

Providence, Rhode Island 02912

${ }^{28} \mathrm{Mg}(\mathrm{B}) *,{ }^{91}{ }_{Y(0)}$

23 Burroughs Corporation 460 Sierra Madra Villa Pasadena, California 91109

$85_{\mathrm{Kr}}(0)$

24 Burroughs Corporation 16701 W. Bernardo Drive San Diego, California 92127

$85_{\mathrm{Kr}}(0)$

25 California Institute of Technology 1201 E. California Boulevard Pasadena, California 91109

$235_{U(0)}$ 
California, University of, see University of California

26 Case-Western Reserve University

2232 Circle Drive

Cleveland, Ohio 44106

${ }^{3} H(0),{ }^{28_{M g}}(8) *, \operatorname{TgS}(0)$

27 Charlotte Memorial Hospital

Nuclear Medicine

1000 Blythe Boulevard

Charlotte, North Carolina 28234

${ }^{127} \times e(B)^{*}$

Chicago, University of, see University of Chicago

28 Children's Hospital

Radiopharmacy

300 Longwood Avenue

Boston, Massachusetts 02115

${ }^{81} \mathrm{Rb}(\mathrm{B})$

29 Cincinnati General Hospital - FDA Nuclear Medicine Laboratory

234 Goodman Street

Cincinnati, Ohio 45267

${ }^{52} \mathrm{Fe}(8)$

30 Colorado State University

Fort Collins, Colorado 80521

${ }^{85} \mathrm{Kr}(0)$

31 Columbia University

Black Building

168th and Ft. Washington Avenue

New York, New York 10032

$81_{\mathrm{Kr}(8)}$

Connecticut, University of, see University of Connecticut

32 Continental Oil Company

Research and Development Department P.O. Box 1267

Ponca City, Oklahoma 74601

${ }^{89} \mathrm{Sr}(0)$
33 Cook Electric Company

6201 0akton Street

Morton Grove, Illinois 60053

$85 \mathrm{Kr}(0)$

34 Cooper Medical Center

Division of Nuclear Medicine

6 th and Stevens Street

Camden, New Jersey 08103

$127 \mathrm{xe}(8)$ *

35 Cornell University

Division of Nutritional Sciences

M.V.R. Hall

Ithaca, New York 14853

${ }^{47} \mathrm{Ca}(0),{ }^{127} \mathrm{Xe}(\mathrm{B})$ *

36 Creighton University

2410 California

Omana, Nebraska 68131

${ }^{47} \mathrm{Ca}(0)$

37 Cryogenic Rare Gas Laboratories, Inc. 730 S. 13th Street Newark, New Jersey 07103

${ }^{85} \mathrm{Kr}(0)$

38 Cyclotron Corporation

950 Gilman Street

Berkeley, California 94710

${ }^{3} \mathrm{H}(0)$

39 DCA Reliability Laboratory 975 Benicia Avenue Sunnyvale, California 94086

$85 \mathrm{Kr}(0)$

40 Delco Electronics

General Motors Corporation

kokomo, Indiana 46901

${ }^{85} \mathrm{Kr}(0)$

41 Department of Energy - Idaho

Health Services Laboratory

550 2nd Street

Idaho Falls, Idaho 83401

${ }^{147} \mathrm{Nd}(\mathrm{I})$ 
Department of Energy

9800 Cass Avenue

Argonne, Illinois 60439

$\mathrm{Pu}(\mathrm{P})$

43 Department of Interior

Geological Survey

National Center

12201 Sunrise Valley Drive

Reston, Virginia 22092

${ }^{252} \mathrm{Cf}(\mathrm{S})$

44 Department of Law Enforcement

Bureau of Identification

Joliet, Illinois 60432

$\operatorname{RSI}(A)$

45 Diagnostic Isotopes

225 Bel leville Avenue

Bloomfield, New Jersey 07003

$127 \times e(8)$ *

46 Duke University

Durham, North Carolina 27706

${ }^{252} \mathrm{Cf}(0), \operatorname{Tgs}(0),{ }^{133} \mathrm{xe}(0)$

du Pont,

see E. I. du Pont de Nemours and Company

47 Eberline Instrument Corporation

P.O. Box 279

Santa Fe, New Mexico 87501

${ }^{250} \mathrm{Cf}(0),{ }^{236} \mathrm{Pu}(0), \mathrm{SS}(0)$

48 Edgerton, Germeshausen and Grier

P.O. Box 98

Goleta, California 93017

$M C P(0)$

49 Edinboro State College

Edinboro, Pennsy 1vania 16444

${ }^{252} \mathrm{Cf}(\mathrm{S})$

50 Edsel B. Ford Institute for Medical

Research

Department of Physics and Biophysics

Henry Ford Hospital

2799 West Grand Boulevard

Detroit, Michigan 48202

${ }^{252} \mathrm{Cf}(\mathrm{S})$
51 EG\&G Idaho, Inc.

P.O. Box 1625

Idaho Falls, Idaho 83401

${ }^{108} \mathrm{Ag}(\mathrm{I}),{ }^{110} \mathrm{Ag}$ ( I),${ }^{28} \mathrm{Al}$ ( I),${ }^{198} \mathrm{Au}(\mathrm{I})$,

${ }^{80} \mathrm{Br}(\mathrm{I}),{ }^{82} \mathrm{Br}(\mathrm{I}),{ }^{47} \mathrm{Ca}(\mathrm{I}),{ }^{49} \mathrm{Ca}(\mathrm{I})$,

${ }^{117} \mathrm{Cd}(\mathrm{I}),{ }^{143} \mathrm{Ce}(\mathrm{I}),{ }^{51} \mathrm{Cr}$ (I), ${ }^{53} \mathrm{Cr}$ (I),

$\mathrm{EU}(\mathrm{P}),{ }^{59} \mathrm{Fe}(\mathrm{I}),{ }^{42} \mathrm{~K}$ (I), ${ }^{140} \mathrm{La}$ (I),${ }^{56} \mathrm{Mn}$ (I),

${ }^{99} \mathrm{Mo}(\mathrm{I}),{ }^{24} \mathrm{Na}$ ( I), $\mathrm{Nd}(\mathrm{I}),{ }^{149} \mathrm{Nd}(\mathrm{I})$,

${ }^{151} \mathrm{Nd}(\mathrm{I}),{ }^{142} \mathrm{Pr}(\mathrm{I}),{ }^{122} \mathrm{Sb}(\mathrm{I}),{ }^{124} \mathrm{Sb}$ (I),

${ }^{77} \mathrm{Se}$ ( I), ${ }^{79} \mathrm{Se}$ ( I), ${ }^{81} \mathrm{Se}$ ( I), ${ }^{153} \mathrm{Sm}$ ( I)

52 E. I. Oupont de Nemours \& Co.

Savannah River Plant

Aiken, South Carolina 29801

${ }^{252} \mathrm{Cf}(0),{ }^{248} \mathrm{Cm},(0),{ }^{137} \mathrm{Cs}(0),{ }^{239}(\mathrm{M})$, $M C P(0),{ }^{238} \mathrm{Pu}(\mathrm{M}),{ }^{236} \mathrm{Pu}(0),{ }^{242} \mathrm{Pu}(0)$,

${ }^{244} \mathrm{Pu}(0), S S(0), \operatorname{Tgs}(0),{ }^{233} U(0)$

53 Electronic Test Center 2200 Walsh Avenue

Santa Clara, California 95050

$85 \mathrm{Kr}(0)$

54 Exxon Nuclear Co., Inc.

2101 Horn Rapids Road

Richland, Washington 99352

$D U(P), E U(P)$

55 Fairchild Semiconductor

P.0. Box 790

Mountain View, California 94040

${ }^{85} \mathrm{Kr}(0)$

56 Fairchild Seimconductors

333 Western Avenue

So. Portland, Maine 04106

$85 \mathrm{Kr}(0)$

57 Florida State University

Tallahassee, Florida 32306

${ }^{252} \mathrm{Cf}(0)$

58 Ford Aerospace and Communication Corporation Division of Ford Motor 3939 Fabian Way

Palo Alto, California 94303

$85_{\mathrm{Kr}}(0)$ 
Gamma Industries

9320 Travenor

P.O. Box 34526

Houston, Texas 77034

${ }^{137} \mathrm{Cs}(0), \operatorname{ss}(0)$

60 Gamma Industries, Inc.

P.0. Box 2543

Baton Rouge, Louisiana 70821

$192 \operatorname{Ir}(0), \mathrm{RW}(0)$

61 Gammie Nuclear Company

11941 Waveland Avenue

Franklin Park, Illinois 60131

$\operatorname{RSI}(A)$

62 Gatan, Inc.

3117 Babcock Boulevard

Pittsburgh, Pennsylvania 15237

${ }^{58} \mathrm{Co}(\mathrm{H}),{ }^{54} \mathrm{Mn}(\mathrm{H})$

63 General Electric Company

P.0. Box 254

San Jose, California 95103

${ }^{241} \mathrm{Am}(0), \operatorname{Cap}(0),{ }^{137} \operatorname{Cs}(0), \operatorname{MCP}(0)$,

Tgs $(0),{ }^{234} \mathrm{U}(0),{ }^{235} \mathrm{U}(0)$

64 General Electric Company

Neutron Devices Department

Pinellas Peninsula Plant

St. Petersburg, Florida 33733

$$
238 \mathrm{Pu}(M)
$$

65 General Electric Company

Vallecitos Nuclear Center

Pleasanton, California 94566

${ }^{58} \mathrm{Co}(\mathrm{H}),{ }^{56} \mathrm{Mn}(\mathrm{H}),{ }^{122} \mathrm{Sb}(\mathrm{I}),{ }^{124} \mathrm{Sb}(\mathrm{I})$

66 General Electric Company

General Electric Drive

Waynesboro, Virginia 22980

${ }^{85} \mathrm{Kr}(0)$

Geological Survey,

see United States Geological Survey
67 Georgia Institute of Technology

225 North Avenue, N.W.

Atlanta, Georgia 30332

$M C P(0),{ }^{90} \mathrm{Sr}(0)$

68 George Washington University

23rd and Eye St. NW

Washington, 0. C. 20037

$77 \mathrm{Br}(\mathrm{L})$

69 Gollob Analytical Service Corporation 47 Industrial Road Berkely Heights, New Jersey 07922

${ }^{3} H(0)$

70 Goodyear Atomic Corp.

P.O. Box 628

Piketon, Ohio 45661

$\mathrm{EU}(\mathrm{P}),{ }^{237} \mathrm{~Np}(0),{ }^{99} \mathrm{Tc}(0)$

71 Gulf Energy and Environmental Systems

P.0. Box 608

San Diego, California 92112

${ }^{140} \mathrm{Ba}(0),{ }^{236} \mathrm{Pu}(0),{ }^{103} \mathrm{Ru}(0),{ }^{95} \mathrm{Zr}(0)$

72 Harry F. Truman Memorial Veterans Hospital

Chief Supply Service

800 Stadium Road

Columbia, Missouri 65201

$127 \times e(B)^{*}$

73 Harvard Medical School

Department of Radiology

50 Binney Street

Boston, Massachusetts 02115

$211_{\text {At (B) }}$

74 Herbert Lehman College of the

City University of New York

Bedford Park Boulevard West

Bronx, New York 10468

${ }^{151} \operatorname{Sm}(0)$

75 Holy Family Hospital

N. 5633 Lidgerwood Street

Spokane, Washington 99207

$133 \mathrm{xe}(0)$ 
76 Honeywell, Inc.

Bldg. 502 Mail Station $\mathbb{M N} 30$ 2540/Tyin City

New Brighton, Minnesota 55112

OU $(P)$

77 Honeywell, Inc. 600 Second St., N.E.

Hopk ins, Minnesota 55343

OU(P)

78 Hughes Aircraft Company

P.O. Box 92907

Los Angeles, California 90009

$85 \mathrm{Kr}(0)$

79 ICN-NB CO

26201 Miles Road

Cleveland, Ohio 44128

$\operatorname{CSI}(0),{ }^{3} H(0),{ }^{129} I(0),{ }^{33} P(0)$, ${ }^{106} \mathrm{Ru}(0),{ }^{90} \mathrm{Sr}(0)$

80 INTEL Corporation

3065 Bowers Avenue

Santa Clara, California 95051

$85_{\mathrm{Kr}}(0)$

81 Indiana University

630 W. New York Street

Indianapolis, Indiana 46202

${ }^{47} \mathrm{Ca}(0)$

82 Industrial Nuclear Company

1124 Chess Drive

Foster City, California 94404

${ }^{192} \operatorname{Ir}(0), \mathrm{RW}(0)$

83 Intelcom Rad Tech

P.0. Box 80817

San Diego, California 92138

${ }^{239} \mathrm{Pu}(0), \operatorname{Tgs}(0)$

84 Interex Corporation

66 Woerd Avenue

Waltham, Massachusetts 02154

$85_{\mathrm{Kr}}(0)$
85 International Nutronics, Inc.

1237 N. San Antonia Rd.

Palo Alto, California 94303

${ }^{252} \mathrm{Cf}(\mathrm{S})$

86 Iowa State University

Ames, Iowa 50010

${ }^{252} \mathrm{Cf}(0),{ }^{129} \mathrm{I}(0), \mathrm{MCP}(0), \mathrm{SS}(0)$

87 Iowa State University

Ames Laboratory

Ames, Iowa 50011

$\operatorname{RSI}(\mathrm{A})$

88 Isotope Products Laboratories

404 South Luke Street

Burbank, California 91502

${ }^{241_{A m}(0),}{ }^{109} \mathrm{Cd}(B) \star,{ }^{252} \mathrm{Cf}(0),{ }^{244} \mathrm{Cm}(0)$, ${ }^{137} \mathrm{Cs}(0), \operatorname{CSI}(0),{ }^{85} \mathrm{Kr}(0),{ }^{237} \mathrm{~Np}(0)$, ${ }^{147} \mathrm{Pm}(0),{ }^{238} \mathrm{Pu}(0),{ }^{240} \mathrm{Pu}(0),{ }^{106} \mathrm{Ru}(0)$, ${ }^{90} \mathrm{Sr}(0), \operatorname{Tgs}(0),{ }^{127} \mathrm{Xe}(\mathrm{B}) *,{ }^{9} 1_{Y}(0)$, ${ }^{95} \operatorname{Zr}(0)$

89 Isovac Engineering Company

6220 San Fernando Road

Glendale, California 91204

${ }^{85} \mathrm{Kr}(0)$

90 J. T. Baker Chemical Company Research Oepartment 600 N. Broad Street Phillipsburg, New Jersey 08865

$\operatorname{RSI}(A)$

91 Jersey Nuclear-Avco Isotopes c/o Exxon Nuclear Company, Inc. 2955 George Washington Way Richland, Washington 99352

Tgs(0), ${ }^{235} \mathrm{U}(0)$

92 Jewish Hospital of St. Louis, The 216 S. Kings Highway Boulevard St. Louis, Missouri 63110

${ }^{47} \mathrm{Ca}(0)$ 
93 John Hopkins Hospital

Radiation Safety

650 N. Wolfe Street

Baltimore, Maryland 21205

${ }^{47} \mathrm{Ca}(0),{ }^{81} \mathrm{Kr}(\mathrm{B}),{ }^{127} \mathrm{Xe}(\mathrm{B})$ *

94 Joint Center for Graduate Study

100 Sprout Road

Richland, Washington 99352

${ }^{54} \mathrm{Mn}(\mathrm{H}),{ }^{24} \mathrm{Na}(\mathrm{H})$

95 KOR Isotopes

56 Rogers Street

Cambridge, Massachusetts 02141

${ }^{3} H(0)$

$96 \quad$ Kaman Nuclear

1700 Garden of the Gods Road

Colorado Springs, Colorado 80907

$3_{H}(0), \operatorname{Tgs}(0)$

97 Knolls Atomic Power Laboratory

P.O. Box 1072

Schenectady, New York 12301

${ }^{243} \mathrm{Am}(0),{ }^{236} \mathrm{Pu}_{\mathrm{u}}(0)$

Kansas, University of, see University of Kansas

Medical Center

Kentucky, University of, see University of Kentucky

98 Lehigh University

Department of Chemistry

Bethlehem, Pennsylvania 18015

${ }^{252} \mathrm{Cf}(\mathrm{S})$

99 Lenoir-Rhyne College

Hickory, North Carolina 38601

${ }^{252} \mathrm{Cf}(\mathrm{S})$

100 Los Alamos Scientific Laboratory

P.O. Box 1663

Los Alamos, New Mexico 87545

$\mathrm{Pu}(\mathrm{P})$
Los Alamos Scientific Laboratory, see University of California Los Alamos Scientific Laboratory

101 Los Angeles County USC Medical Center 1200 N. State Street, Box 112 GH Los Angeles, California 90033

${ }^{47} \mathrm{Ca}(0)$

102 Lovelace Clinic 4800 Gibson Boulevard, S.E. Albuquerque, New Mexico 87108

$\operatorname{MCP}(0),{ }^{233} U(0),{ }^{232} U(0)$

103 Lovelace Foundation of Medicine 5200 Gibson Boulevard, S.E. Albuquerque, New Mexico 87108

$\mathrm{NU}(\mathrm{P})$

104 Mallinckrodt Chemical Works $3600 \mathrm{~N}$. Second Street

St. Louis, Missouri 63160

${ }^{137} \mathrm{Cs}(0),{ }^{152+154} \mathrm{Eu}(0),{ }^{127} \mathrm{Xe}(\mathrm{B}) \star$, $133 \mathrm{Xe}(0)$

Mallinckrodt Institute of Radiology, see Washington University School of Medicine

105 Mary Hitchcock Memorial Hospital Radiation Therapy Department Hanover, New Hampshire 03755

$\operatorname{MCP}(0)$

Maryland, University of, see University of Maryland

106 Massachusetts General Hospital 32 Fruit Street Boston, Massachusetts 02114 ${ }^{47} \mathrm{Ca}(0),{ }^{18} \mathrm{~F}(\mathrm{~B}),{ }^{82} \mathrm{Sr}(\mathrm{L}),{ }^{85} \mathrm{Sr}(\mathrm{L})$

107 Massachusetts Institute of Technology Building 20

Cambridge, Massachusetts 02139

124 Sb (B) 
108 Mayo Foundation

Rochester, Minnesota 55901

$47 \mathrm{Ca}(0),{ }^{99} \mathrm{TC}(0),{ }^{127} \mathrm{Xe}(\mathrm{B})^{*}$

109 Memorial Hospital

Department of Nuclear Medicine

1400 East Boulder

Colorado Springs, Colorado 80909

$133 \times$ e (0)

110 Merck and Company

Rahway, New Jersey 07065

${ }^{60} \mathrm{Co}(0)$

111 Methodist Hospital

Nuclear Medicine

1604 N. Capitol Avenue

Indianapolis, Indiana 46202

$127 \mathrm{Xe}(\mathrm{B})$ *

112 Methodist Hospital

6720 Bertner

Houston, Texas 77025

$133 \mathrm{xe}(0)$

113 Michael-Reese Hospital

29th Street and Ellis Avenue Chicago, Illinois 60616

133 xe $(0)$

Michigan, University of, see University of Michigan

114 Mine Safety Appliance Company 210 North Braddock Avenue Pittsburgh, Pennsylvania 15208

131 I(0)

115 Mine Safety Appliances Company $R 02$

Evans City, Pennsylvania 16033

131 I (0)

116 Mini-Dose Laboratories, Inc.

1825 Emerson Street

Denver, Colorado 80218

$133 \mathrm{xe}(0)$
117 Minnesota Mining and Manufacturing Company

P.O. Box 3121

St. Paul, Minnesota 55101

${ }^{137} \mathrm{Cs}(0),{ }^{85} \mathrm{Kr}(0), \operatorname{MCP}(0),{ }^{95} \mathrm{Nb}(0)$, ${ }^{103} \mathrm{Ru}(0)$

118 Minnesota Mining and Manufacturing Company

Twin Cities Army Ammunition Plant Building 590

New Brighton, Minnesota 55112

$210 p_{0}(M), 127 X_{e}(B)$ *

Mississippi, University of, see University of Mississippi

119 Monsanto Research Corporation

Dayton Laboratory

1515 Nicholas Road

Dayton, Ohio 45407

${ }^{241_{A m}(0), C a p(0),}{ }^{252} \mathrm{Cf}(\mathrm{S}),{ }^{244} \mathrm{Cm}(0)$

120 Monsanto Research Corporation Mound Facility

P.O. Box 32

Miamisburg, Ohio 45342

${ }^{144} \mathrm{Ce}(0),{ }^{137} \mathrm{Cs}(0), \operatorname{MCP}(0),{ }^{106} \mathrm{Ru}(0)$, $95 \mathrm{Zr}(0)$

121 Moravek Biochemicals 15302 E. Proctor Avenue City of Industry, California 91745 ${ }^{3} H(0)$

122 Motorola, Inc. 5005 East McDowe 11 Road Phoenix Arizona 85008

${ }^{85} \mathrm{Kr}(0)$

NASA, see National Aeronautics and Space Administration

123 N. L. Industries, Inc. 1130 Central Avenue Albany, New York 12205

DU(P) 
124 Nassau County Medical Center Meadowbrook Hospital 2201 Hempstead Turnpike East Meadow, New York 11554 ${ }^{47} \mathrm{Ca}(0)$

125 National Aeronautics and Space Administration Lewis Research Center 21000 Brookpark Road Cleveland, Ohio 44135 $3_{H(0)}$

National Aeronautics and Space Administration

Goddard Soace Flight Center Glendale Road

Greenbelt, Maryland 20771

${ }^{153} \mathrm{Gd}(0),{ }^{85} \mathrm{Kr}(0), \mathrm{ss}(0)$

127 National Aeronautics and Space Admin istration

Ames Research Center

Moffett Field, California 94035

${ }^{47} \mathrm{Ca}(0)$

128 National Animal Disease Center ARS, Building \#1, Room 121

R. R. 2, Dayton Avenue

Ames, lowa 50010

${ }^{28} \mathrm{Mg}(\mathrm{B})$ *

129 National Bureau of Standards

Rec. Room, Building 301

Route 270 and Quince Orchard Rd.

Gaithersburg, Maryland 20760

${ }^{238} \mathrm{Pu}(\mathrm{M}),{ }^{127} \mathrm{Xe}(\mathrm{B})$ *

130 National Bureau of Standards

Connecticut Avenue and Van Ness Street, N.W.

Washington, D.C. 20234

${ }^{140} \mathrm{Ba}(0),{ }^{252} \mathrm{Cf}(0),{ }^{237} \mathrm{~Np}(0), \operatorname{Tgs}(0)$, $235 \mathrm{U}(0),{ }^{133} \mathrm{Xe}(0)$

131 National Institutes of Health

Building 21

Bethesda, Maryland 20014

${ }^{47} \mathrm{Ca}(0),{ }^{43} \mathrm{~K}(0),{ }^{91} \mathrm{Y}(0),{ }^{127} \mathrm{Xe}(\mathrm{B}) *$,
132 National Lead of Ohio

P.O. Box 39158

Cincinnati, Ohio 45239

$D U(P), E U(P)$

133 National Naval Medical Center Radiation Safety Service Building \#144

Bethesda, Maryland 20014

${ }^{127} \mathrm{xe}(\mathrm{B})$ *

134 National Semiconductor Corporation 2900 Semiconductor Drive

Santa Clara, California 95051

$85 \mathrm{Kr}(0)$

135 Naval Regional Medical Center Receiving Officer, Building 505 8750 Mountain Boulevard Oakland, California 94627

${ }^{47} \mathrm{Ca}(0),{ }^{127} \times \mathrm{e}(\mathrm{B})$ *

Navy, see United States Navy

Nebraska, University of, see University of Nebraska

136 New England Medical Center Hospitals 151 Harrison Avenue Boston, Massachusetts 02111

${ }^{89} \operatorname{Sr}(0)$

137 New England Nuclear Corporation 575 Albany Street Boston, Massachusetts 02118

${ }^{144} \mathrm{Ce}(0),{ }^{137} \mathrm{Cs}(0), \operatorname{CSI}(0),{ }^{153} \mathrm{Gd}(0)$, $3_{H(0)},{ }^{129} \mathrm{I}(0),{ }^{85} \mathrm{Kr}(0), \operatorname{MCP}(0)$, ${ }^{63} \mathrm{Ni}(0),{ }^{33} \mathrm{P}(0),{ }^{147} \mathrm{Pm}(0),{ }^{103} \mathrm{Ru}(0)$,

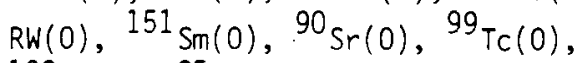
${ }^{133} \mathrm{Xe}(0),{ }^{95} \mathrm{Zr}(0)$,

138 New England Nuclear Corporation 601 Treble Cove Rd.

North Billerica, Massachusetts 01862

${ }^{109} \mathrm{Cd}(\mathrm{B}) *,{ }^{68} \mathrm{Ge}(\mathrm{B}){ }^{22} \mathrm{Na}(\mathrm{B}) *$, $127 \mathrm{Xe}(\mathrm{B})$ * 
New Orleans, University of, see University of New Orleans

New York, State University of see State University of New York

139 New York University Medical Center 550 First. Avenue

New York, New York 10016

$233 \mathrm{u}(0), 235 \mathrm{U}(0)$

140 North Carolina Baptist Hospital Nuclear Medicine 300 S. Hawthorne

Winston-Salem, North Carolina 27103

$127 \mathrm{Xe}(\mathrm{B})$ *

141 North Carolina Memorial Hospital Division of Nuclear Medicine Chapel Hill, North Carolina 27514

${ }^{123} \mathrm{I}(\mathrm{B}), \quad{ }^{127} \mathrm{Xe}(\mathrm{B})$ *

142 North Shore University Hospital Department of Nuclear Medicine 300 Community Drive Manhasset, New York 10030 125 I(B)

143 North Texas State University P.O. Box 13767

N. T. Station

Denton, Texas 76203

${ }^{252} \mathrm{Cf}(0)$

144 Northwestern Memorial Hospital Nuclear Medicine 239 E. Chicago Avenue Chicago, Illinois 60611 $127 \mathrm{xe}(\mathrm{B})$ *

145 Northwestern University 619 Clark Street Evanston, Illinois 60201

${ }^{129} I(0), \operatorname{MCP}(0)$

146 Norwalk Hospital Nuclear Medicine 24 Stevens Street Norwalk, Connecticut 06850

$127 \mathrm{Xe}(\mathrm{B})$ *
147 Nuclear Dynamics 2614 Seaman Avenue El Monte, California 91733

${ }^{241} \mathrm{Am}(0)$

148 Nuclear Environmental Engineering, Inc. P.O. Box 58866 Houston, Texas 77058

${ }^{241_{A m}(0),}{ }^{137} \mathrm{Cs}(0),{ }^{3} \mathrm{H}(0),{ }^{192} \operatorname{Ir}(0)$, ${ }^{85} \mathrm{Kr}(0), \operatorname{MCP}(0), \mathrm{RW}(0)$

149 Nuclear Fuel Services, Incorporated Erwin, Tennessee 37650

${ }^{99}$ Tc (0)

150 Nuclear Materials, Inc. 2229 Main Street Concord, Massachusetts 01742

$\operatorname{DU}(P)$

151 Nuclear Radiation Development, Inc. 2937 A1t Boulevard Grand Island, New York 14072

${ }^{241}$ Am (0)

Nuclear Sources and Services

P.O. Box 14023

Houston, Texas 77021

$241_{A m(0)}$

153 Nuc 1ide Corporation 642 East College Avenue State College, Pennsylvania 16801

238 U(M)

154 Oak Ridge Associated Universities P.O. Box 117

Oak Ridge, Tennessee 37830

$\operatorname{CSI}(0), \operatorname{MCP}(0), \operatorname{SS}(0),{ }^{99} \mathrm{TC}(0)$

155 Occidental 0 il Shale Company, Inc. P.O. Box 2999

Grand Junction, Colorado 81501

$85 \mathrm{Kr}(0)$

156 Ohio State University 190 North Oval Drive Columbus, Ohio 43210

${ }^{89} \mathrm{Sr}(0)$ 
157 Ohio State University

Research Foundation

1315 Xinnear Road

Columbus, Ohio 43212

${ }^{153} \mathrm{Gd}(0),{ }^{3} H(0),{ }^{89} \mathrm{Sr}(0), \operatorname{Tgs}(0)$

158 Oklahoma Medical Research Institute 825 N.E. 13th Street

Oklahoma City, Oklahoma 73104

SS $(0)$

Oregon, University of, see University of Oregon

159 Oregon State University Corvallis, Oregon 97330

\section{${ }^{252} \mathrm{Cf}(\mathrm{s})$}

160 Pacific Radiopharmacy 347 North Kuakini Street Honolulu, Hawaii 96817

\section{${ }^{133} \mathrm{xe}(0)$}

161 Palmdale General Hospital Palmdale, California 93550

$133 \mathrm{xe}(0)$

Pennsylvania, University of, see University of Pennsylvania

162 Penrose Hospital 2215 North Cascade Colorado Springs, Colorado 80907

${ }^{133} \mathrm{Xe}(0)$

163 Pleasant Valley Hospital

2309 Antonio Avenue

Camarillo, California 93010

${ }^{133} \mathrm{Xe}(0)$

Pittsburgh, University of, see University of Pittsburgh

164 Purdue University

Chemistry Department

W. Lafayette, Indiana 47907

RSI (A)
165 Rad X Corporation

1390 w. Belt North

Houston, Texas 77043

${ }^{127} \mathrm{Xe}(\mathrm{B}) *$

166 Radiation Resources, Inc.

24 Kulick Road

Fairfield, New Jersey 07006

${ }^{137} \operatorname{Cs}(0), \operatorname{MCP}(0)$

167 Radium Chemical Company, Inc.

161 East 42nd Street

New York, New York 10036

${ }^{3}(0)$

168 Raytheon Company

350 Ellis Street

Mountain View, California 94042

$85 \mathrm{Kr}(0)$

169 Reactor Experiments, Inc.

963 Terminal Way

San Carlos, California 94070

233 U(0)

170 Rensselaer Polytechnic Institute Service Building

Troy, New York 12181

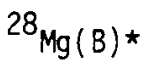

171 Reuter-Stokes E1ectronics Components 18530 South Miles Parkway

Warrensvi 1 le Heights

Cleveland, Ohio 44128

${ }^{234} u(0)$

Rhode Island, University of, see University of Rhode Island

172 Richland Memorial Hospital

Nuclear Medicine

3301 Harden Street

Columbia, South Carolina 29203

$127 \mathrm{Xe}(\mathrm{B})$ *

Rochester, University of, see University of Rochester 
173 Rockwell International

Atomics International Division

Rocky Flats Plant

Rocky Flats, Colorado 80401

$$
\begin{aligned}
& { }^{241} \mathrm{Am}(P), \quad{ }^{243} \mathrm{Am}(0), \quad \mathrm{Du}(\mathrm{P}), \mathrm{Pu}(\mathrm{P}), \\
& 238 \mathrm{Pu}(\mathrm{M}),{ }^{239} \mathrm{Pu}(\mathrm{M}),{ }^{236} \mathrm{Pu}(\mathrm{O}), \mathrm{Tgs}(0)
\end{aligned}
$$

174 Rockwell Hanford Operations

P.0. Box 800

Richland, Washington 99352

${ }^{241} \mathrm{Am}(\mathrm{H}), \mathrm{OU}(\mathrm{P}), \mathrm{Pu}(\mathrm{P}),{ }^{239} \mathrm{Pu}(\mathrm{H})$,

${ }^{238} \mathrm{Pu}(\mathrm{P}), \operatorname{Ra} 0 E F(\mathrm{H}),{ }^{90} \mathrm{Sr} Y(H),{ }^{233} \mathrm{U}(P)$

175 Rush Presbyterian

St, Luke's Medical Center

Nuclear Medicine

1753 W. Congress Parkway

Chicago, Illinois 60612

${ }^{52} \mathrm{Fe}(\mathrm{B}),{ }^{89} \mathrm{Sr}(0)$

San Francisco, University of,

see University of San Francisco

176 Sandia Laboratories

P.O. Box 5800

Alburquerque, New Mexico 87115

${ }^{252} \mathrm{Cf}(0), \mathrm{DU}(\mathrm{P}),{ }^{85} \mathrm{Kr}(0),{ }^{237} \mathrm{~Np}(0)$,

Tc $(0),{ }^{99} \mathrm{Tc}(0), \operatorname{Tgs}(0),{ }^{238} \mathrm{U}(0)$

177 Sandia Laboratories

Kirtland Air Force Base (East)

Receiving Section, Building 895

Albuquerque, New Mexico 87115

${ }^{3} \mathrm{H}(\mathrm{M}), \quad{ }^{238} \mathrm{Pu}(\mathrm{M})$

178 Schlumberger Technology Corporation

old Quarry Road

P.0. Box 307

Ridgefield, Connecticut 06877

${ }^{252} \mathrm{Cf}(\mathrm{S})$

179 Schwarz Bio-Research, Inc. Mountain View Avenue Orangeburg, New York 10962

${ }^{3} H(0)$
180 Scripps Clinic and Research Foundation 476 Prospect Street La Jolla, California 92037

${ }^{89} \mathrm{Sr}(0)$

181 Self-Powered Lighting, Ltd. 8 Westchester Plaza Elmsford, New York 10523

$3_{H(0)}$

182 Shell Development Company 1410 45th Street Emeryville, California 94608

$85_{\mathrm{Kr}}(0)$

183 Shepherd and Associates, J. L. 1614 Victory Boulevard Glendale, California 91201

${ }^{137} \operatorname{Cs}(0), \operatorname{MCP}(0)$

184 Signetics Corporation 1275 South 300 East Orem, Utah 84057

$85 \mathrm{Kr}(0)$

185 Sloan-Kettering Institute 425 East 68th Street New York, New York 10021 $133 \mathrm{Xe}(0)$

186 Source Production and Equipment Co., Inc. 625 0x ley Street Kenner, Louisiana 70062

${ }^{192} \operatorname{Ir}(0), \mathrm{RW}(0)$

South Alabama, University of, see University of South Alabama

South Carolina, University of, see University of South Carolina

Southern California, University of, see University of Southern California

187 Spire Corporation

Patriots Park

Bedford, Massachusetts, 01730

${ }^{60} \mathrm{Co}(0), \operatorname{MCP}(0), \operatorname{Tgs}(0)$ 
188 St. Francis Hospital

Department of Nuclear Medicine

6161 South Yale

Tulsa, Oklahoma 74136

$133 \mathrm{Xe}(0)$

189 St. John's Hospital

333 North F Street

Oxnard, California 93030

$133 \mathrm{xe}(0)$

190 St. John's Mercy Medical Center 615 South New Ballas Road Nuclear Medicine

St. Louis, Missouri 63147

$133 \times e(0)$

191 St. Mary's College

Winona, Minnesota 55987

${ }^{239} \mathrm{Pu}(\mathrm{M})$

192 St. Patrick Hospital

524 South Ryan Street

Lake Charles, Louisiana 70601

133 xe(0)

193 St. Vincent Medical Center

$3 r d$ and Grandview

Los Angeles, California 90057

${ }^{133} \mathrm{xe}(0)$

194 State university of New York

Downstate Medical Center

450 Clarkson Avenue

Brooklyn, New York 11203

${ }^{99} \mathrm{Tc}(0)$

195 State University of New York

Buffalo, New York 14214

${ }^{18} F(B),{ }^{89} \mathrm{Sr}(0)$

196 State University of New York

Research Foundation

Stony Brook, New York 11790

Tgs $(0),{ }^{235} U(0)$
197 Statek Corporation

1200 Alvarez Avenue

Orange, California 92668

$85_{\mathrm{Kr}}(0)$

198 Swedish-American Hospital

1400 Charles Street

Rockford, Illinois 61101

133 xe (0)

199 Syntex Corporation

3401 Hi11view Avenue

Palo Alto, California 94304

${ }^{3} \mathrm{H}(0)$

200 Technetium Corporation

1111 Rose Hill Drive

Charlottesville, Virginia 22901

$\operatorname{Tgs}(0)$

201 Technical Operations

South Avenue

Burlington, Massachusetts 01803

${ }^{137} \operatorname{Cs}(0),{ }^{192} \operatorname{Ir}(0), \operatorname{MCP}(0), \operatorname{RW}(0)$

202 Teledyne Energy Systems

110 West Timonium Road

Tirnonium, Maryland 21093

${ }^{238} \mathrm{Pu}(\mathrm{M})$

203 Teledyne Wah Chang Albany

Division of Teledyne Ind., Inc.

P.O. Box 460

Albany, Oregon 97321

${ }^{23} \mathrm{U}(0)$

204 Tempset, Inc.

1251 Grover Road

St. Louis, Missouri 63125

$85 \mathrm{Kr}(0)$

Tennessee, University of, see University of Tennessee

205 Texas Instruments, Inc. Science Services Division P.O. Box 5012, Mail Stop 6 Dallas, Texas 75222

${ }^{85} \mathrm{Kr}(0)$ 
206 Texas Instruments, Inc.

13588 N. Central Expressway

Dallas, Texas 75243

$238_{U(M)}$

Texas, University of

see University of Texas

207 Texatope Pharmacy

7342 Oak Manor

San Antonio, Texas 78229

${ }^{133} \mathrm{Xe}(0)$

208 Texatope Pharmacy

3911 Tulane Avenue

New Orleans, Louisiana 70119

$133 \mathrm{xe}(0)$

209 Touro Infirmary

Research Department

1401 Foucher Street

New Orleans, Louisiana 70115

${ }^{28} \mathrm{Mg}(\mathrm{B})^{*}$

210 Trio-Tech, Inc.

A Division of Tracer Flo

2435 N. Naomi

Burbank, California 91504

$85_{\mathrm{Kr}}(0), \mathrm{SS}(0)$

211 Union Carbide Corporation

P.0. Box X

Oak Ridge, Tennessee 37830

$241_{\mathrm{Am}}(0),{ }^{243} \mathrm{Am}(0),{ }^{140} \mathrm{Ba}(0)$,

${ }^{249} \mathrm{Bk}(0),{ }^{47} \mathrm{Ca}(0),{ }^{249} \mathrm{Cf}(0),{ }^{244} \mathrm{Cm}(0)$,

${ }^{58} \mathrm{Co}(\mathrm{H}),{ }^{60} \mathrm{Co}(0), \operatorname{CSI}(0),{ }^{137} \mathrm{Cs}(0)$,

$153 \mathrm{Gd}(0),{ }^{3} H(0),{ }^{3} H(P),{ }^{131} \mathrm{I}(0)$,

$85_{\mathrm{Kr}}(\mathrm{O}), \operatorname{MCP}(\mathrm{O}),{ }^{54} \mathrm{Mn}(\mathrm{H}),{ }^{95} \mathrm{Nb}(0)$,

$\mathrm{Np}(\mathrm{P}),{ }^{231} \mathrm{~Pa}(\mathrm{M}), \mathrm{Pu}(\mathrm{P}),{ }^{239} \mathrm{Pu}(0)$,

${ }^{106} \mathrm{Ru}(0),{ }^{151_{S m}(0),}{ }^{89} \mathrm{Sr}(0), \mathrm{SS}(0)$,

${ }^{99} \mathrm{Tc}(0), \operatorname{Tgs}(0),{ }^{229} \mathrm{Th}(\mathrm{M}),{ }^{230} \mathrm{Th}(\mathrm{M})$,

${ }^{234} \operatorname{Th}(M),{ }^{232} \operatorname{Th}(0),{ }^{233} U(0),{ }^{235} U(0)$,

${ }^{236} \mathrm{U}(0),{ }^{238} \mathrm{U}(0),{ }^{133} \mathrm{Xe}(0),{ }^{95} \mathrm{Zr}(0)$

212 Union Carbide Corporation

Nuclear Division

Oak Ridge Gaseous Diffusion Plant, K-25

Oak Ridge, Tennessee 37830

238 U( M)
213 Union Carbide Corporation,

Nuclear Division

Oak Ridge Gaseous Diffusion Plant

Post Office Box P

Oak Ridge, Tennessee 37830

${ }^{85} \mathrm{Kr}(0),{ }^{230} \mathrm{Th}(0),{ }^{133} \mathrm{Xe}(0)$

214 Union Carbide Corporation

Nuclear Division Y-12 Plant

P.O. Box Y

Oak Ridge, Tennessee 37830

${ }^{241} \mathrm{Am}(0), \operatorname{CSI}(0), \mathrm{DU}(P), \operatorname{EU}(P),{ }^{153} \mathrm{Gd}(0)$, $S S(0), \operatorname{Tgs}(0),{ }^{235} U(M),{ }^{233} U(0)$, ${ }^{235} U(0){ }^{238} U(0)$

215 Unit Process Assemblies, Inc. 53-15 37th Avenue

Woodside, New York 11377

$147 \mathrm{Pm}(0),{ }^{90} \mathrm{Sr}(0)$

216 UNC Nuclear Industries, Inc.

P.O. Box 490

Richland, Washington 99352

${ }^{241} \mathrm{Am}(\mathrm{H}),{ }^{133} \mathrm{Ba}(\mathrm{H}),{ }^{144} \mathrm{CePr}(\mathrm{H})$,

${ }^{109} \mathrm{Cd}(\mathrm{H}),{ }^{57} \mathrm{Co}(\mathrm{H}),{ }^{134} \mathrm{Cs}(\mathrm{H})$,

${ }^{137} \mathrm{Cs}(H), \mathrm{DU}(\mathrm{P}), \mathrm{EU}(\mathrm{P}),{ }^{54} \mathrm{Mn}(\mathrm{H})$,

${ }^{106} \mathrm{Ru}(\mathrm{H}),{ }^{85} \mathrm{Sr}(\mathrm{H}), \operatorname{Th}(\mathrm{P}),{ }^{65} \mathrm{Zn}(\mathrm{H})$

217 United States Air Force Academy

USAF Academy, Colorado 80840

${ }^{252} \mathrm{Cf}(\mathrm{S})$

218 United States Army

Aberdeen Proving Ground

Ballistics Research Laboratory

Aberdeen Proving Ground, Maryland 21005

DU $(P)$

219 United States Amy

Aberdeen Proving Ground

Materiel Command

Aberdeen Proving Ground, Maryland 21005

$\mathrm{DU}(\mathrm{P})$ 
220 United States Amy

Letterman Medical Center

Finance and Accounting Division

Building 102

Presidio of San Francisco, California

94129

${ }^{47} \mathrm{Ca}(0),{ }^{133} \times \mathrm{e}(0)$

221 United States Department of Energy Chicago Operations Office

Finance Division

9800 South Cass Avenue

Argonne, Illinois 60439

${ }^{252} \mathrm{Cf}(0),{ }^{85} \mathrm{Kr}(0), \mathrm{SS}(0)$

222 United States Department of Energy

Richland Operations Office

P.O. Box 550

Richland, Washington 99352

$243_{\mathrm{Am}}(0), 152+154_{\mathrm{Eu}(0)},{ }^{236} \mathrm{Pu}(0)$,
${ }^{95} \mathrm{Zr}(0)$

For other listings for the

United States Department of Energy,

see Department of Energy

United States Department of Interior, see Department of Interior

223 United States Geological Survey

Financial Management

National Center, Room 1447-A

12201 Sunrise Valley Drive

Reston, Virginia 22092

${ }^{243} \mathrm{Am}(0),{ }^{144} \mathrm{Ce}(0),{ }^{244} \mathrm{Cm}(0)$,

224 United States Navy

Ammunition Depot

Crane, Indiana 47522

$85 \mathrm{Kr}(0)$

225 United States Public Health Service

P.0. Box 684

4505 Maryland Parkway

Las Vegas, Nevada 89101

${ }^{230} \mathrm{Th}(0)$
226 United States Public Health Service Hospital

15th Avenue and Lake Street

San Francisco, California 94118

${ }^{47} \mathrm{Ca}(0)$

227 United States Radium Corporation 4150 Old Berwick Road

P.O. Box 380

Bloomsburg, Pennsylvania 17815

$3_{H(0)}$

228 United States Testing Company, Inc. 2800 George Washington Way

Richland, Washington 99352

${ }^{241} \mathrm{Am}(\mathrm{H})$,
${ }^{239} \mathrm{Pu}(\mathrm{H}),{ }^{147} \mathrm{Pm}(\mathrm{H})$,

$U$ (natural)(H)

229 United Technologies Corporation Silver Lane

East Hartford, Connecticut 06108

Cap (0), ${ }^{244} \mathrm{Cm}(0)$

230 University of Alabama

Medical Center

1919 Seventh Avenue, South

Birmingham, Alabama 35486

133 Xe $(0)$

231 University of Arizona

Post Office Drawer 4100

Tucson, Arizona 85721

$M C P(0)$

University of Arkansas

Fayettevi1le, Arkansas 72701

${ }^{252} \mathrm{Cf}(\mathrm{S})$

233 University of California

Lawrence Berkeley Laboratory

University of California

Berkeley, California 94720

${ }^{249} \mathrm{Bk}(0),{ }^{248} \mathrm{Cm}(0), \mathrm{Pu}(\mathrm{P}),{ }^{82} \mathrm{Sr}(\mathrm{L})$, 
234 University of California

Davis, California 95616

${ }^{129} I(0)$

235 University of California

Post Office Box 109

La Jolla, California 92037

${ }^{144} \mathrm{Ce}(0),{ }^{127} \mathrm{Xe}(\mathrm{B})$ *

236 University of California - San Diego

Chemistry Department

3175 Miramar Road

La Jolla, California 92093

$\operatorname{RSI}(\mathrm{A})$

237 University of California

Nuclear Medicine Department

3175 Miramar Road

La Jolla, California 92093

${ }^{82} \operatorname{Sr}(L),{ }^{85} \mathrm{Sr}(L)$

238 University of California

Lawrence Livermore Laboratory

P.O. Box 808

Livermore, California 94551

$241_{\mathrm{Am}}(0),{ }^{243} \mathrm{Am}(0),{ }^{249} \mathrm{Bk}(0)$,

${ }^{244} \mathrm{Cm}(0),{ }^{248} \mathrm{Cm}(0),{ }^{253} \mathrm{Es}(0)$,

$257 \mathrm{Fm}(0),{ }^{3} \mathrm{H}(0), \mathrm{MCP}(0),{ }^{239} \mathrm{Pu}(0)$,

${ }^{244} \mathrm{Pu}(0), \mathrm{Pu}_{u}(0), S S(0), \operatorname{Tg} s(0),{ }^{233} \mathrm{U}(0)$,

$235 v(0)$,

239 University of California

900 Veteran Avenue

Los Angeles, California 90024

${ }^{243} \mathrm{Am}(0),{ }^{244} \mathrm{Cm}(0),{ }^{133} \mathrm{Xe}(0)$

240 University of California

Radiology Department

650 Circle Drive, South

Los Angeles, California 90024

${ }^{52} \mathrm{Fe}(\mathrm{L})$

241 University of California

Purchasing Department

Orange, California 92668

${ }^{90} \mathrm{Sr}(0)$
242 University of California

University Hospital Rec.

225 W. Dickinson Street

San Diego, California 92103

$127 \mathrm{xe}(\mathrm{B})$ *

243 University of California

San Francisco Medical Center

San Francisco, California 94122

${ }^{252} \mathrm{Cf}(\mathrm{S}),{ }^{133} \mathrm{Xe}(0)$

244 University of California Los Alamos Scientific Laboratory Los Alamos, New Mexico 87544

${ }^{241} \operatorname{Am}(0), \operatorname{Cap}(0),{ }^{249} \mathrm{Cf}(0),{ }^{252} \mathrm{Cf}(0)$, ${ }^{252} \mathrm{Cf}(\mathrm{S}),{ }^{243} \mathrm{Cm}(\mathrm{O}),{ }^{248} \mathrm{Cm}(\mathrm{O}),{ }^{58} \mathrm{Co}(\mathrm{H})$, ${ }^{253} \mathrm{Es}(0),{ }^{56} \mathrm{Mn}(\mathrm{H}),{ }^{239} \mathrm{Pu}(\mathrm{M}),{ }^{238} \mathrm{Pu}(\mathrm{M})$, ${ }^{240} \mathrm{Pu}(0),{ }^{244} \mathrm{Pu}(0), \mathrm{SS}(0), \operatorname{Tgs}(0)$, ${ }^{236} U(0),{ }^{238} U(0)$

245 University of Chicago 5641 S. Ingleside Avenue Chicago, Illinois 60637

RSI (A)

246 University of Chicago 950 E. 59th Street Chicago, Illinois 60637

${ }^{252} \mathrm{Cf}(\mathrm{S}),{ }^{82} \mathrm{Sr}(\mathrm{L}),{ }^{85} \mathrm{Sr}(\mathrm{L})$

247 University of Chicago Office of the Comptroller 1525 E. Hyde Park Boulevard Chicago, Illinois 60615

${ }^{47} \mathrm{Ca}(0),{ }^{89} \mathrm{Sr}(0)$

248 University of Chicago 1160 East 55th Street Chicago, Illinois 60615

${ }^{47} \mathrm{Ca}(0),{ }^{89} \mathrm{Sr}(0)$

249 University of Connecticut Physiology Department

Farmington, Connecticut 06032

${ }^{28} \mathrm{Mg} \mathrm{(B)}$ * 
250 University of Kansas Medical Center Department of Diagnostic Radiation 39 th and Rainbow Boulevard Kansas City, Kansas 66103 ${ }^{77} \mathrm{Br}(\mathrm{L}),{ }^{47} \mathrm{Ca}(0){ }^{127} \mathrm{Xe}(\mathrm{B})$ *

251 University of Kentucky Lexington, Kentucky 40506

${ }^{252} \mathrm{Cf}(\mathrm{S}),{ }^{3} \mathrm{H}(0), \mathrm{MCP}(0),{ }^{28} \mathrm{Mg}(\mathrm{B})$ *

252 University of Loyola Medical Center 2160 S. 1st Avenue

Maywood, lllinois 60153

$\operatorname{CSI}(A)$

253 University of Maryland Baltimore County Campus 5401 Wilkins Drive Baltimore, Maryland 21228

${ }^{106} \mathrm{Ru}(0)$

254 University of Michigan

Ann Arbor, Michigan 48104

${ }^{252} \mathrm{Cf}(\mathrm{S}),{ }^{237} \mathrm{~Np}(0),{ }^{99} \mathrm{Tc}(0), \operatorname{Tgs}(0)$, $233 \mathrm{U}(0)$

255 University of Mississippi

Jackson, Mississippi 39216

$43_{K}(0),{ }^{28} \mathrm{Mg}(B)$ *

256 University of Nebraska

511 Administration

Lincoln, Nebraska 68508

$232 \cup(0)$

257 University of New Orleans

Lakefront

New Orleans, Louisiana 70122

$\operatorname{CSI}(0)$

258 University of Oregon

Oregon Medical School

3181 S. W. Sam Jackson Park Road

Portland, Oregon 97201

$133 \mathrm{xe}(0)$
259 University of Pennsylvania

3025 Walnut Street

Philadelphia, Pennsylvania 19104

${ }^{252} \mathrm{Cf}(0), \operatorname{Tgs}(0),{ }^{133} \mathrm{Xe}(0)$

260 University of Pennsylvania Hospital

Radiology Department

3400 Spruce Street

Phi ladelphia, Pennsylvania 19104

${ }^{18} \mathrm{~F}(\mathrm{~B}),{ }^{123} \mathrm{I}(\mathrm{B})$

261 University of Pittsburgh

Pittsburgh, Pennsylvania 15213

${ }^{243} \mathrm{Am}(0),{ }^{236} \mathrm{Pu}(0)$

262 University of Rhode Island Narrangansett Marine Laboratory Kingston, Rhode Island 02881

${ }^{140} \mathrm{Ba}(0),{ }^{137} \mathrm{Cs}(0)$

263 University of Rochester

575 Elmwood Avenue

Rochester, New York 14627

${ }^{242} \mathrm{Pu}(0), \operatorname{Tgs}(0)$

264 University of San Francisco Golden Gate and Parker Avenues San Francisco, California 94117

${ }^{237} \mathrm{~Np}(0), \operatorname{Tgs}(0),{ }^{235_{U}(0)}$

265 University of South Alabama Nuclear Medicine Laboratory 2451 Sillingim Mobile, Alabama 36688

${ }^{28} \mathrm{Mg}(\mathrm{B})$ *

266 University of South Carolina Columbia, South Carolina 29208

${ }^{106} \mathrm{Ru}(0)$

267 University of Southern California University Park Los Angeles, California 90007

$M C P(0)$ 
University of Southern California School of Pharmacy

1985 Zonal Avenue

Los Angeles, California 90033

${ }^{252} \mathrm{Cf}(\mathrm{S})$

269 University of Tennessee

Knoxville, Tennessee 37916

${ }^{192} \operatorname{Ir}(0),{ }^{28} \mathrm{Mg}(8) *,{ }^{238} \mathrm{U}(0)$

270 University of Tennessee-DOE

Agriculture Research Laboratory

1299 Bethel Valley Road

Oak Ridge, Tennessee 37830

${ }^{95} \mathrm{Nb}(0)$

271 University of Texas

Austin, Texas 78712

${ }^{129} \mathrm{I}(0)$

272 University of Texas

Nuclear Medicine Service

Room 229, Clinical Science Building

Galveston, Texas 77550

${ }^{47} \mathrm{Ca}(0),{ }^{127} \mathrm{Xe}(\mathrm{B}) *,{ }^{133} \mathrm{Xe}(0)$

273 University of Texas at Dallas

P.0. Box 30365

Dallas, Texas 75230

${ }^{47} \mathrm{Ca}(0)$

274 University of Utah

Purchasing Department

Salt Lake City, Utah 84112

$241_{\mathrm{Pu}}(0), \quad{ }^{244} \mathrm{Pu}(0),{ }^{133} \mathrm{Xe}(0)$

275 University of Virginia Charlottesville, Virginia 22901

${ }^{99} \mathrm{TC}(0),{ }^{127} \times \mathrm{e}(\mathrm{B})$ *

276 University of Virginia Hospital

Charlottesville, Virginia 22901

${ }^{153} \mathrm{Gd}(0), \mathrm{SS}(0),{ }^{127} \mathrm{Xe}(\mathrm{B})$ *
277 University of Washington

400 Administration Building

Seattle, Washington 98105

${ }^{244} \mathrm{Cm}(0),{ }^{129} \mathrm{I}(0),{ }^{244} \mathrm{Pu}(0),{ }^{238} \mathrm{Pu}(0)$,

${ }^{239} \mathrm{Pu}(0),{ }^{240} \mathrm{Pu}(0),{ }^{89} \mathrm{Sr}(0), \operatorname{Tgs}(0)$,

${ }^{133} \mathrm{Xe}(0)$

278 University of Wisconsin

750 University Avenue

Madison, Wisconsin 53706

${ }^{153} \mathrm{Gd}(0),{ }^{3} \mathrm{H}(0), \mathrm{SS}(0)$

Utah, Unitersity of, see University of Utah

279 Veterans Administration

Data Processing Center

1615 Woodward Street East

Austin, Texas 78722

$\operatorname{MCP}(0),{ }^{133} \mathrm{Xe}(0)$

280 Veterans Administration Center Wood, Wisconsin 53193

$43 \times(0)$

281 Veterans Administration Hospital 700 South 19th Street

Birmingham, Alabama 35233

${ }^{99} \mathrm{Tc}(0),{ }^{133} \mathrm{Xe}(0)$

282 Veterans Administration Hospital Chief Supply Service

11201 Benton Street

Loma Linda, California 92354

$127 \mathrm{xe}(\mathrm{B})$ *

283 Veterans Administration Hospital Wilshire and Sawtelle Boulevards Los Angeles, California 90073

47

$\mathrm{Ca}(0)$

284 Veterans Administration Hospital 1055 Clerrnont Street

Denver, Colorado 80220

$133 \mathrm{xe}(0)$ 
Veterans Administration Hospital Chief Supply Service

1201 North West 16th Street

Miami, Florida 33125

133

xe(0)

286 Veterans Administration Hospital Chief Supply Service

Building \#7

Hines, Illinois 60141

${ }^{47} \mathrm{Ca}(0),{ }^{252} \mathrm{Cf}(\mathrm{S}),{ }^{28} \mathrm{Mg}(\mathrm{B})$ *

287 Veterans Administration Hospital 1310 24th Avenue S.

Nashville, Tennessee 37203

$133 \times \mathrm{xe}(0)$

288 Veterans Administration Hospital 1400 VFW Parkway

W. Roxbury, Massachusetts 02132

${ }^{123} \mathrm{I}(\mathrm{B}),{ }^{127} \mathrm{Xe}(\mathrm{B})$ *

289 Veterans Administration Hospital Nuclear Medicine

Northport, New York 11768

$127 \mathrm{Xe}(\mathrm{B})$ *

290 Veterans Administration Hosp it a 1 Nuclear Medicine NM-115

4435 Beacon Avenue South

Seattle, Washington 98108

${ }^{103} \mathrm{Ru}(\mathrm{B})$

291 Vanderbilt University

Radiation Safety

Room 2, v0217

Nashvi1le, Tennessee 37232

$127 \mathrm{Xe}$

Virginia, University of, see University of Virginia

292 Vought Corporation

P.O. Box 6144

Dallas, Texas 75222

${ }^{252} \mathrm{Cf}(\mathrm{s})$

Washington, University of, see University of Washington
293 Washington University

Lindell and Skinker

St. Louis, Missouri 63130

${ }^{47} \mathrm{Ca}(0),{ }^{131} \mathrm{I}(0),{ }^{133} \mathrm{Xe}(0)$

294 Washington University

Department of Radiology

499 South Euclid

St Louis, Missouri 63110

${ }^{77} \mathrm{Br}(\mathrm{L})$

295 Washington University School of Medicine Mallinkrodt Institute of Radiology

510 South Kings Highway

St. Louis, Missouri 63110

${ }^{252} \mathrm{Cf}(\mathrm{s})$

296 Western Electric Company

P.O. Box 241

Reading, Pennsylvania 19603

$85 \mathrm{Kr}(0)$

297 Western Electric Company, Inc.

P.0. Box 1409

Lawrence, Massachusetts 01842

$85 \mathrm{Kr}(0)$

298 Western Michigan University

Kalamazoo, Michigan 49001

${ }^{3} H(0), \operatorname{Tgs}(0)$

299 Westinghouse Electric Corporation Bettis Atomic Power Laboratory P.O. Box 79

West Mifflin, Pennsylvania 15122

$\operatorname{Tgs}(0),{ }^{232} \operatorname{Th}(0),{ }^{233} \mathrm{U}(0),{ }^{235} \mathrm{U}(0)$

300 Westinghouse Electric Corporation P.O. Box 284

Elmira, New York 14902

${ }^{234} \mathrm{u}(0),{ }^{238} \mathrm{u}(0)$

301 Westinghouse Electric Corporation Advanced Reactors Division

P.O. Box 158

Madison, Pennsylvania 15663

${ }^{243} \mathrm{Am}(0),{ }^{244} \mathrm{Cm}(0)$ 
302 Westinghouse Hanford Company

Hanford Engineering Development

Laboratory

P.O. Box 1970

Richland, Washington 99352

$241_{\mathrm{Am}}(\mathrm{O}),{ }^{133} \mathrm{Ba}(\mathrm{H}),{ }^{144} \mathrm{CePr}(\mathrm{H})$,

${ }^{252} \mathrm{Cf}(\mathrm{O}),{ }^{252} \mathrm{Cf}(\mathrm{P}),{ }^{244} \mathrm{Cm}(\mathrm{H})$,

${ }^{134} \mathrm{Cs}(H),{ }^{137} \mathrm{Cs}(H), \operatorname{CSI}((0), \mathrm{DU}(P)$,

$\mathrm{EU}(\mathrm{P}),{ }^{85} \mathrm{Kr}(\mathrm{O}), \operatorname{MCP}(0),{ }^{22} \mathrm{Na}(\mathrm{H})$,

$\left.{ }^{237} \mathrm{~Np}(\mathrm{H}),{ }^{237} \mathrm{~Np} 0\right), \mathrm{Np}(P) \mathrm{NU}(P), P_{u}(P)$,

$\operatorname{Th}(P),{ }^{233} U(P),{ }^{90} \mathrm{Sr} Y(H),{ }^{147} \mathrm{Pm}(H)$,

$\left.\left.239 \mathrm{Pu}(\mathrm{H}), \quad{ }^{240} \mathrm{Pu} \mathrm{H}\right), \quad 236 \mathrm{Pu} 0\right)$,

$\left.\left.{ }^{239} \mathrm{Pu}(0),{ }^{240} \mathrm{Pu}, 0\right),{ }^{241} \mathrm{Pu} .0\right)$,

${ }^{242} \mathrm{Pu}(0),{ }^{106} \mathrm{Ru}(0), \mathrm{SS}(0), \operatorname{Tgs}(0)$,

${ }^{230} \operatorname{Th}(0),{ }^{232} \operatorname{Th}(0),{ }^{233} \mathrm{U}(0),{ }^{234} \mathrm{U}(0)$,

${ }^{235} \mathrm{u}(0), 238 \mathrm{U}(0)$

Westinghouse Research and Development Center

1310 Beulah Road, Churchill Boro

Pittsburgh, Pennsylvania 15235

${ }^{58} \mathrm{Co}(\mathrm{H}),{ }^{54} \mathrm{Mn}(\mathrm{H})$
304 Wi11iam Beaumont Hospital 3601 W. Thirteen Mile Road Royal Oak, Michigan 48072

$133 \mathrm{xe}(0)$

Wisconsin, University of, see University of Wisconsin

305 Woods Hole Oceanographic Institute Woods Hole, Massachusetts 02543

$\mathrm{Sp}(0),{ }^{229} \operatorname{Th}(0),{ }^{236} \mathrm{U}(0)$

306 Yale-New Haven Hospital

Diagnostic Radiology and Nuclear Medicine

333 Cedar Street

New Haven, Connecticut 06510

$81_{K r(B)}$

307 Yale University

20 Ashmun Street

New Haven, Connecticut 06520

${ }^{129} \mathrm{I}(0)$ 
B. FOREIGN CUSTOMERS

$\underline{\text { ARGENTINA }}$

308 Comision Nacional de Energia Atomica Gerencia de Energia

Avda. del Libertador 8250

Buenos Aires, Argentina

$233 U(0)$

\section{$\underline{\text { AUSTRALIA }}$}

309 Australian Atomic Energy Comission Research Establishment

Lucas Heights, Australia

${ }^{244} \mathrm{Cm}(0), \operatorname{Tgs}(0),{ }^{127} \mathrm{Xe}(\mathrm{B})$ *

310 Australian Radiation Laboratory 36 Lonsdale Street

Melbourne, Victoria, 3000 Australia

$127 \mathrm{xe}(\mathrm{B}){ }^{*}$

311 Embassy of Australia

1601 Massachusetts Avenue, N.W.

Washington, D.C. 20036

${ }^{243} \mathrm{Am}(0), 242 \mathrm{Pu}(0),{ }^{234} \mathrm{U}(0)$

312 Union Carbide/Australia, Ltd.

157-167 Liverpool Street

Sidney, Australia

$127 \times e(B)$

\section{$\underline{\text { AUSTRIA }}$}

313 International Atomic Energy Agency

Kaertnerring 11-13

A-1010 Vienna, Austria

$$
\begin{aligned}
& { }^{241} \mathrm{Am}(0),{ }^{237} \mathrm{~Np}(0), \operatorname{Tgs}(0),{ }^{232} \mathrm{Th}(0), \\
& 233_{U(0)},{ }^{235} \mathrm{U}(0),{ }^{238} \mathrm{U}(0)
\end{aligned}
$$

\section{$\underline{\text { BELGIUM }}$}

314 Centre d'Etude de I'Energie Nucleaire 144 Avenue Flasky

Brussels 4, Belgium

${ }^{237} \mathrm{~Np}(0),{ }^{239} \mathrm{Pu}(0), \operatorname{Tgs}(0),{ }^{235} \mathrm{U}(0)$, $238 \mathrm{U}(0)$
315 EURATOM

51-53 Rue 8elliard

Brussels, Belgium

${ }^{236} \mathrm{Pu}(0),{ }^{240} \mathrm{Pu}(0),{ }^{241} \mathrm{Pu}(0)$, ${ }^{242} \mathrm{Pu}(0)$

316 European Atomic Energy Commission Central Bureau for Nuclear Measurements Geel, Belgium

${ }^{239} \mathrm{Pu}(0),{ }^{242} \mathrm{Pu}(0), \quad 233 \mathrm{u}(0)$

317 Katholieke Universiteit Leuven Dienst Radioprotektie

De Croylaan 54

Heverlee, Belgium

${ }^{28} \mathrm{Mg}(\mathrm{B})$ *

318 Universite de Liege

2 rue Armand Stevart

Liege, Belgium

Cap (0), ${ }^{237} \mathrm{~Np}(0)$

319 IRE, Institut National des Radioelements Avenue Eugene Plasky, 144 1040 Brussels, Belgium

$85 \mathrm{Kr}(0)$

CANADA

Alberta, University of, see University of Alberta

320 Atomic Energy of Canada Limited Chalk River, Ontario, Canada

${ }^{3} H(M),{ }^{235} U(0)$

321 Atomic Energy of Canada Limited P.O. Box 6300

Postal Station J

Ottawa, Canada

${ }^{137} \operatorname{Cs}(0), \operatorname{MCP}(0)$

322 Atomic Energy of Canada Limited Whiteshell Nuclear Research Establishment Pinawa, Manitoba, Canada

${ }^{230} \operatorname{Th}(0)$ 
British Columbia, University of, see University of British Columbia

323 Health Sciences Center

Nuclear Medicine

700 Wi 11 iam Avenue

Winnipeg, Canada

$127 \mathrm{Xe}(\mathrm{B})$ *

Manitoba, University of, see University of Manitoba

324 MeGill University

Montreal 2, P.0., Canada

133 xe(0)

325 McMaster University

Hamilton, Ontario, Canada

${ }^{129} \mathrm{I}(0)$

326 McMaster University Medical Center Forsyth Avenue Receiving

Hamilton, Ontario, Canada

$127 \mathrm{xe}(8)^{*}$

327 Mitel Semiconductor, Inc.

18 Airport Boulevard

Bromont, Quebec

Canada JOE 1 LO

$85_{\mathrm{Kr}}(0)$

328 Oueen's University

Biology Department

Kingston, Ontario, Canada

${ }^{28} \mathrm{Mg}(\mathrm{B}) *,{ }^{127} \mathrm{Xe}(\mathrm{B})$ *

329 Simon Fraser University

Burnaby 2, British Columbia, Canada

${ }^{129} \mathrm{I}(0)$

Toronto, University of, see University of Toronto

330 Toronto General Hospital 101 College Street Toronto 2, Ontario, Canada

$133 \times \mathrm{ee}(0)$
331 University Hospital

Nuclear Medicine Department

London, Ontario, Canada

$127 \mathrm{xe}(\mathrm{B})$ *

332 University of Alberta

Edmonton, Alberta, Canada

${ }^{252} \mathrm{Cf}(0),{ }^{3} \mathrm{H}(0), \operatorname{Tgs}(0),{ }^{133} \mathrm{Xe}(0)$

333 University of British Columbia Vancouver 8, British Columbia, Canada

235 u(0)

334 University of Manitoba

Department of Animal Science

Winnipeg, Canada

${ }^{252} \mathrm{Cf}(0),{ }^{28} \mathrm{Mg}(\mathrm{B}) *,{ }^{127} \mathrm{Xe}(\mathrm{B})$ *

335 University of Toronto

Toronto, Canada

${ }^{252} \mathrm{Cf}(0),{ }^{28} \mathrm{Mg}(8)$ *

336 Vancouver General Hospital Division of Nuclear Medicine Vancouver, British Columbia, Canada

${ }^{127} \mathrm{Xe}(\mathrm{B}) *,{ }^{133} \mathrm{Xe}(0)$

DENMARK

337 Isotope Pharmacy

378 Freder iksundsvej

Broenshoej, Denmark

$127 \mathrm{xe}(8)$ *

ENGLAND

338 Brandhurst Co. Ltd.

Wellington Road

High Wycombe, Bucks HP12 3PS

Engl and

$3_{H(0)}$

339 Edlow International Co. Suite 404-5

1100 17th Street, N.W.

Washington, D.C. 20036

$3_{H(0)}$ 
Mal 1 inckrodt, Ltd.

Building 521 129G

Southampton Road

Houns low, Middlesex, England

$127 \mathrm{xe}(\mathrm{B})$ *

Oxford, University of,

see University of Oxford

341 Radiochemical Centre

White Lion Road

Amersham, Bucks, England

Cap (0), ${ }^{144} \mathrm{Ce}(0),{ }^{244} \mathrm{Cm}(0),{ }^{153} \mathrm{Gd}(0)$, ${ }^{3} \mathrm{H}(0),{ }^{85} \mathrm{Kr}(0), \mathrm{MCP}(0),{ }^{147} \mathrm{Pm}(0)$, ${ }^{89} \mathrm{Sr}(0),{ }^{99} \mathrm{Tc}(0),{ }^{91_{Y}(0),},{ }^{95} \operatorname{Zr}(0)$

342 Sanders-Roe Developments, Ltd. North Hyde Road

Hayes, Middlesex, England

$3_{H}(0)$

343 United Kingdom

Atomic Energy Research Establishment

Didcot, Oxfordshire

England OXON, OX11, ORA

$E U(P),{ }^{242} \mathrm{Pu}(0),{ }^{234} \mathrm{U}(0),{ }^{235} \mathrm{U}(0)$,

$238 \mathrm{U}(0)$

344 United Kingdom Treasury and Supply Delegation

25 Broadway

New York, New York 10004

$3^{3}(0)$

345 University of Oxford

Nuclear Physics Laboratory

21 Banbury Road

Oxford, England

${ }^{244} \mathrm{Cm}(0), \operatorname{Tgs}(0)$

346 V. G. Micro Mass

Nat Lane

Winsforn, Cheshire, England

${ }^{3} H(M)$
FRANCE

347 CEA

Department des Radioelements

B.P. No. 2

91190 Gif Sur Yvette, France

$140_{\mathrm{Ba}(0)},{ }^{153} \mathrm{Gd}(0), \mathrm{SS}(0)$

348 French Atomic Energy Commission 1730 Rhode Island Avenue, N.W.

Suite 1217

Washington, D.C. 20036

Cap (0), ${ }^{238} \mathrm{Pu}(0),{ }^{242} \mathrm{Pu}(0)$

\section{GERMANY, FEDERAL REPUBLIC OF}

349 American Hoechst Corporation

P.O. Box 2500

Somerville, New Jersey 08876

$85 \mathrm{Kr}(0)$

$350 \quad B$ iolog ische Ansta1t He 1goland Laboratorium Sulldorf

Wustland 2, 2000 Hamburg 55

Federal Republic of Germany

${ }^{244} \mathrm{pu}(0)$

351 EURATOM Transuranium Institute

P.O. Box 452

75 Karlsruhe

Federal Republic of Germany

${ }^{242} \mathrm{Pu}(0),{ }^{230} \mathrm{Th}(0),{ }^{233} \mathrm{U}(0)$

352 Freie Universitat

1 Berlin 33

Federal Republic of Germany

${ }^{129}$ I (0)

GWK-Gese 11schaft zur Wiederaufberitung von Kernt

D-7051 Leopoldshafen,

Federal Republic of Germany

${ }^{242} \mathrm{Pu}(0)$

354 Gesellschaft fur Kernforschung mBH

Institut fur Angewandte Reaktor Physik

Weverstrasse 5

75 Karlsruhe

Federa 1 Repub 1i c of Germany

Cap (0), ${ }^{238} \mathrm{Pu}(0),{ }^{241} \mathrm{Pu}(0)$, 238 U(0) 
355 Gesellschaft. fur Schwerionenforschunp mBH Target labor

Postfach 541

0.6100 Darmstadt

Federal Republic of Germany

${ }^{236} \mathrm{Pu}(0), \operatorname{Tgs}(0),{ }^{238} \mathrm{U}(0)$

356 Hahn-Meitner Institut

fur Kernforschung Berlin, Sekton Kernphysick

Berlin,

Federal Republic of Germany

${ }^{99} \mathrm{Tc}(0)$

357 Johann Wolfgang Goethe University Frank furt/Main

Federal Republic of Germany

$236 \mathrm{U}(0)$

358 Kernforschung Sanlage

Postfach 365

517 Julich

Federal Republic of Germany

$237 \mathrm{~Np}(0), \operatorname{Tgs}(0)$

359 Medizinische Hochschule Hannover Dept. of Radioloqie

Karl-Wiechert-Allee 9

Hannover,

Federal Republic of Germany

${ }^{127} \mathrm{Xe}(8)$ *

360 Technischen Hochschule Munchen

Arcistrasse 21

8 Munich 2

Germany

${ }^{241} \mathrm{Am}(0), \operatorname{Cap}(0),{ }^{244} \mathrm{Cm}(0),{ }^{237} \mathrm{~Np}(0)$

361 University of Erlangen-Nurnberg

Gluchstr.

852 Erlangen

Federal Republic of Germany

${ }^{252} \mathrm{Cf}(0)$

362 University of Mainz/Rhein

Mainz am Rhein

Federal Republic of Germany

${ }^{236} \mathrm{Pu}(0)$
363 University of Munster

44 Munster/Westfalln

Federal Republic of Germany

$37 \operatorname{Ar}(0)$

HONG KONG

364 National Cash Register Mfg, Co., Ltd.

No. 55 Wong Chuk Hang Road

Aberdeen, Hong Kong

$85 \mathrm{Kr}(0)$

365 Semi-Conductor Devices, Ltd.

59-61 Wong Chuk Hang Road

Aberdeen, Honq Kong

$85_{\mathrm{Kr}(0)}$

$\underline{\text { ISRAEL }}$

366 Landseas Corporation

1212 Avenue of the Americas

New York, New York 10036

${ }^{237} \mathrm{~Np}(0), \operatorname{Tgs}(0)$

367 Packard Instrument, Ltd.

16 Oholian Street

Jerusalem, Israel

$28 \mathrm{Mg}(\mathrm{B})$ *

JAPAN

368 International Antex, Inc. 2630 California Street Mountain View, California 94040

MCP (0), ${ }^{147} \mathrm{Pm}(0)$

369 Japan Radioisotope Association Hon-Komagome 2 Chome 28

8unkyo-Ku

Tokyo, Japan

$\operatorname{MCP}(0),{ }^{147} \mathrm{Pm}(0)$

370 Mitsus and Company (USA), Inc.

200 Park Avenue

New York, New York 10017

234 u(0) 
371 Reactivity Accident Laboratory JAERI

Tokai-Mura, Ibaraki-Ken

Japan

$\varepsilon U(P)$

\section{NETHERLANDS}

372 N. V. Philips-Duphar

P.O. Box 2

Weesp, The Netherlands

$\operatorname{csI}(0)$

NEW ZEALANO

$$
\begin{aligned}
& 373 \text { University of Auckland } \\
& \text { Private Bag } \\
& \text { Auck } 1 \text { and, New Zealand } \\
& 106_{\text {Ru(0) }}
\end{aligned}
$$

NORWAY
374 Institute for Atomic Energy Isotope Laboratories
$\mathrm{N}-2007$
Kjeller, Norway
$127 \mathrm{xe}(\mathrm{B})$ *

375 Institutt for Atom Energi OECD Halden Reactor Project Halden, Norway

$E U(P)$

\section{$\underline{\text { SCOTLAND }}$}

376 University of Glasgow

Medical Cardiology

Royal Infirmary

Glasgow, Scotland

\section{${ }^{28} \mathrm{Mg}(\mathrm{B})$ *}

SWEDEN

377 University of Uppsala

Uppsala, Sweden

$\operatorname{Tgs}(0), \quad 235 \mathrm{U}(0)$

\section{SWITZERLAND}

378 Eidg. Institut for Reaktorforschung Wurenlingen, Switzerland

${ }^{242} \mathrm{Pu}(0),{ }^{99} \mathrm{Tc}(0)$

379 Merz and Bentelli AG

Bump 1 izstrasse 91

3018 Bern, Switzerland

$3_{H(0)}$

380 Radium-Chemie

A. Zeller and Company

9053 Teufen/Ar

Switzerland

${ }^{3} H(0), \operatorname{MCP}(0),{ }^{147} P_{m}(0)$

TAIWAN

381 United Pacific Semiconductor Corporation 1, San-Chin Street

Su-Lin Cheng, Taipei, Hsien, Taiwan

$85 \mathrm{Kr}(0)$ 


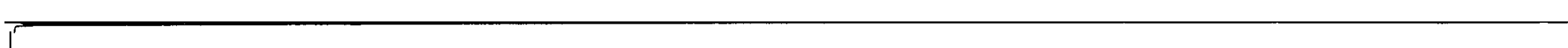




\section{A. DOMESTIC}

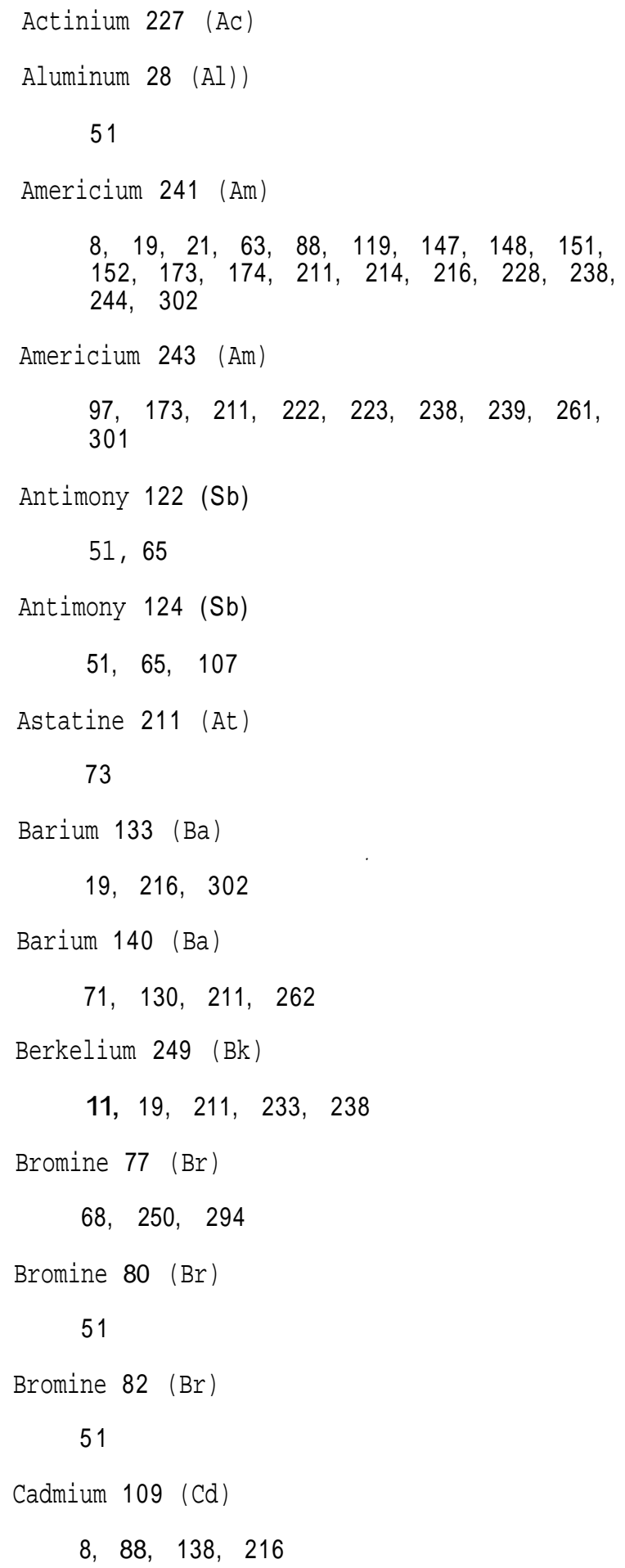

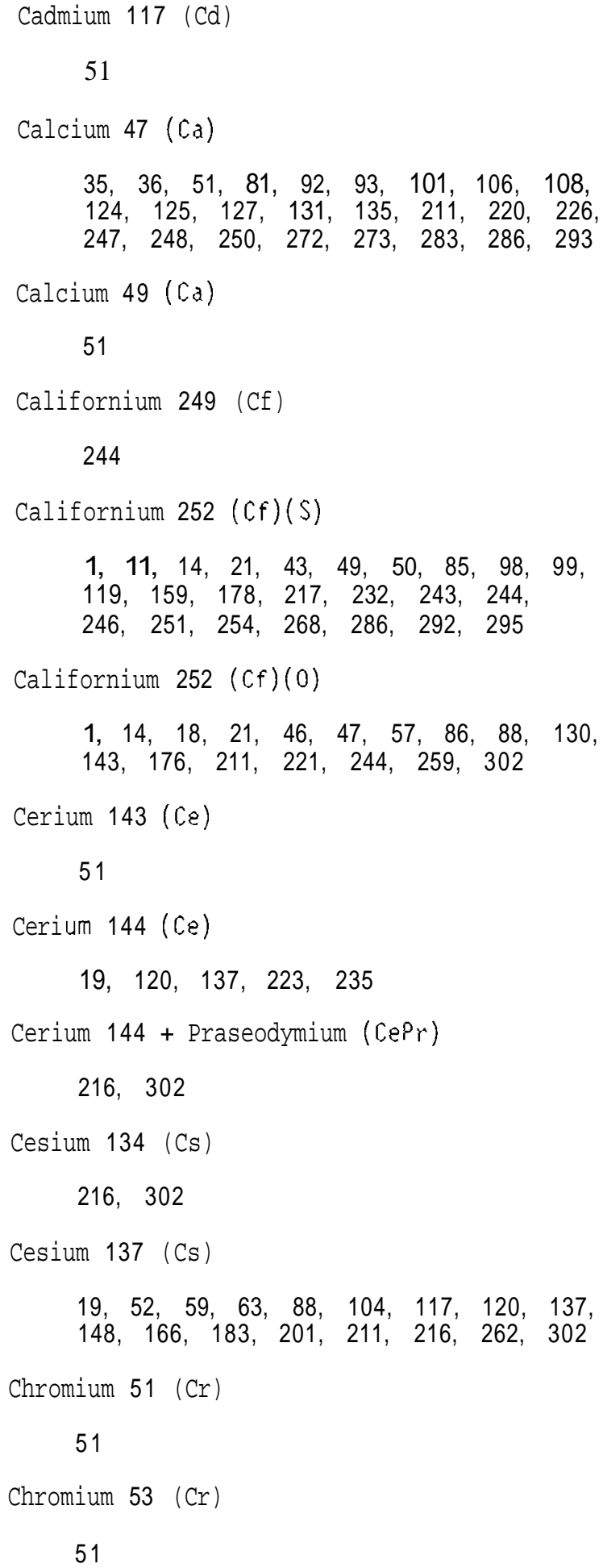




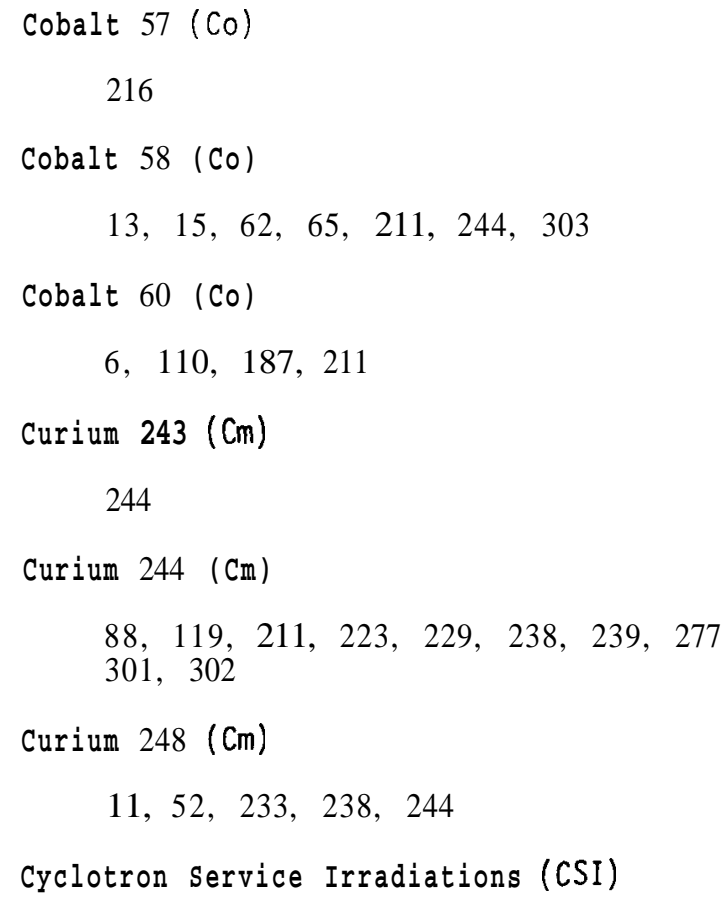

Gold 198 (Au)

6,51

Hydrogen $3(H)$

$7,8,19,21,26,38,69,79,95,96$,

$121,125,137,148,157,167,177$.

$179,181,199,211,227,238,251$, 278,298

Iodine 123 (I)

$141,260,288$

Iodine 125 (I)

142

Iodine 129 (I)

$8,79,86,137,145,234,271,277$, 307

Iodine 131 (I)

$114,115,211,293$

Iridium 192 (Ir)

16, 60, 82, 148, 186, 201, 269

Iron 52 (Fe)

$29,175,240$

Iron 55 (Fe)

13

Iron 59 (Fe)

51

Krypton $81(K r)$

4, 31, 93, 306

Krypton $85(\mathrm{Kr})$

$1,3,5,7,19,23,24,30,33,37$, $39,40,53,55,56,58,66,78,80$, $84,88,89,117,122,126,134,137$, $148,155,168,176,182,184,197,204$, $205,210,211,213,221,224,296,297$, 302

Lanthanum 140 (La)

51

Magnesium $28(\mathrm{Mg})$

$22,26,128,170,209,249,251,255$, $265,269,286$ 
Manganese $54(\mathrm{Mn})$

$13,62,94,211,216,303$

Manganese $56(\mathrm{Mn})$

$13, \quad 15,51,65,244$

Miscellaneous Compound Preparation (MCP)

$20,48,52,63,67,86,102,105,117$, $120,137,145,148,154,166,183,187$,

$201,211,231,238,251,267,279,302$

Molybdenum 99 (Mo)

51

Neodymium (Nd)(I)

51

Neodymium $147(\mathrm{Nd})$

41

Neodymium $149(\mathrm{Nd})$

51

Neodymium $151(\mathrm{Nd})$

51

Neptunium (Np)

$11,211,302$

Neptunium 237 (Np)

$11,17,70,88,130,176,223,254$, 264,302

Nickel $63(\mathrm{Ni})$

137

Niobium $95(\mathrm{Nb})$

$11,19,117,211,270$

Phosphorus $33(P)$

$79, \quad 137$

Plutonium ( $\mathrm{Pu}$ )

$42,100,173,174,211,233,238,302$

Plutonium 236 (Pu)

$1,11,18,19,47,52,71,97,173,222$, $223, \quad 228, \quad 261, \quad 302$
Plutonium 238 (Pu)

$10,19,21,52,64,88,129,173,174$, $177, \quad 202, \quad 244, \quad 277$

Plutonium 239 (Pu)

19, 83, 173, 174, 191, 211, 228, 238, $244, \quad 277, \quad 302$

Plutonium 240 (Pu)

$88,244,277,302$

Plutonium 241 (Pu)

$274, \quad 302$

Plutonium 242 (Pu)

$11,52,263,302$

Plutonium 244 (Pu)

$17, \quad 19,21, \quad 52,238,244, \quad 274,277$

Polonium 210 (Po)

$118, \quad 228$

Potassium 42 ( $K$ )

51

Potassium $43(K)$

131, 255, 280

Praseodymium $142(\mathrm{Pr})$

51

Promethium 147 (Pm)

$19,88,137,215,228,302$

Protactinium $231(\mathrm{~Pa})$

19, 211

RaDEF

19, 174

Reactor Service Irradiations (RSI)

$9,44,61,87,90,164,236,245$

Rubidium 81 (Rb)

28

Ruthenium 103 (Ru)

21, 71, 117, 137, 290 


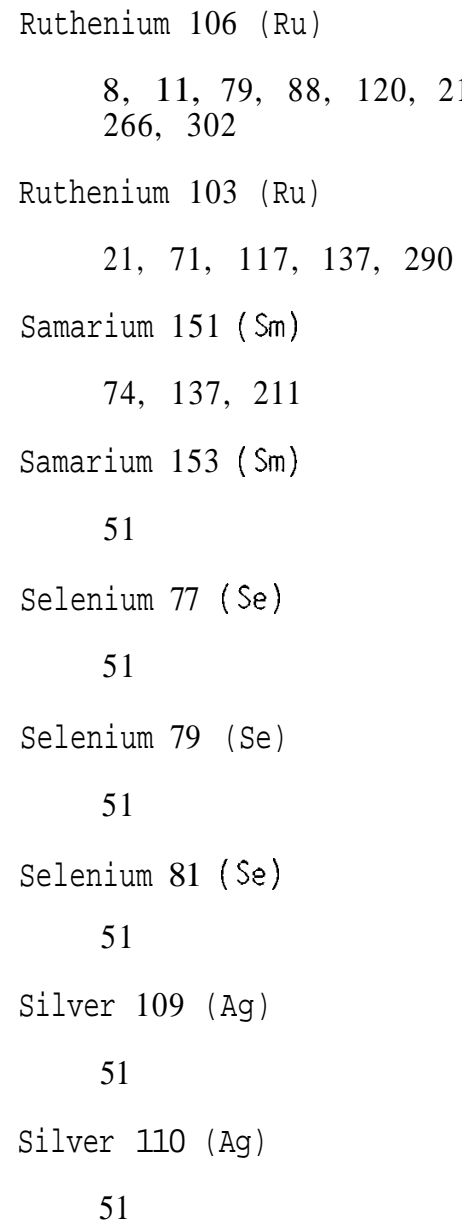

Strontium 90 (Sr)

$67,79,88,137,215,241$

Strontium 90 - Yttrium (SrY)

19, 174, 228, 302

Tantalium 182 (Ta)

13,15

Target Preparation, Radioisotope (Tgs)

$1,8,11,14,17,19,21,26,46,52$, $63,83,88,91,96,130,157,173,176$, $187,196,200,211,214,238,244,254$, $259,263,264,277,298,299,302$

Technetium ( TC)

176

Technetium 99 (TC)

$11,19,70,108,137,149,154,176,194$ $211,233,254,275,281$

Thorium 229 (Th)

211,305

Thorium 230 (Th)

$11,211,213,225,302$

Thorium 232 (Th)

$11,21,211,299,302$

Thorium 234 (Th)

211

Thorium $(T h)(P)$

216, 302

Thulium 170 (Tm)

19

Uranium-Depleted (DU)

$2,11,54,76,77,123,132,150,173$,

$174,176,214,216,218,219,302$

Uranium-Enriched (EU)

$51,54,70,132,214,216,302$

Uranium-Natural (RW)

$18,19,103,228,302$ 
Uranium 232 (U)

$18, \quad 102, \quad 256$

Uranium 233 (U)

$11,17,52,102,139,169,174,203$, $211,214,238,254,299,302$

Uranium 234 (U)

$63, \quad 171,233,300,302$

Uranium 235 (U)

$11,25,63,91,130,139,196,211,214$, $238, \quad 264, \quad 299,302$

Uranium 236 (U)

$211, \quad 244, \quad 305$

Uranium 238 (U)

$7,11,17,153,176,206,211,212$, $244, \quad 269,300,302$

Withdrawn Radioisotopes (RW)

$16,60,82,137,148,186,201$

Xenon 127 (Xe)

$12,27,34,35,45,72,88,93,104$, $108,111,118,129,131,133,135,138$, $140,141,144,146,165,172,235,242$, $250,272,275,276,282,288,289,291$

Xenon 128 (Xe)

12

Xenon 133 (Xe)

$1,3,46,75,104,109,112,113,116$, $130,137,160,161,162,163,185,188$, $189, \quad 190,192,193,198,207,208,211$, $213,220,230,239,243,258,259,272$, $274,277,279,281,284,285,287,293$, 304

Yttrium $91(Y)$

$11,22,88,131$

Zinc $65(\mathrm{Zn})$

216

Zirconium $95(\mathrm{zr})$

$1,11,19,71,88,120,137,211,222$
B. FOREIGN

Americium 241 (Am)

$313, \quad 360$

Americium 243 (Am)

311

Argon 37 (Ar)

363

Barium 140 (Ba)

347

Californium 252 (Cf)(0)

$332, \quad 334, \quad 335, \quad 361$

Cerium 144 (Ce)

341

Cesium 137 (Cs)

321

Curium $244(\mathrm{~cm})$

$309,341,345,360$

Cyclotron Service Irradiations (CSI)

372

Encapsulation (Cap)

$318,341,348,354,360$

Gadolinium 153 (Gd)

$341, \quad 347$

Hydrogen $3(H)$

$320,332,338,339,341,342,344$, $346, \quad 379, \quad 380$

Iodine 129 (I)

$325, \quad 329, \quad 352$

Krypton 85 ( $\mathrm{Kr})$

$319, \quad 327,341,364,365,381$

Magnesium $28(\mathrm{Mg})$

$317,328,334,335,367,376$ 


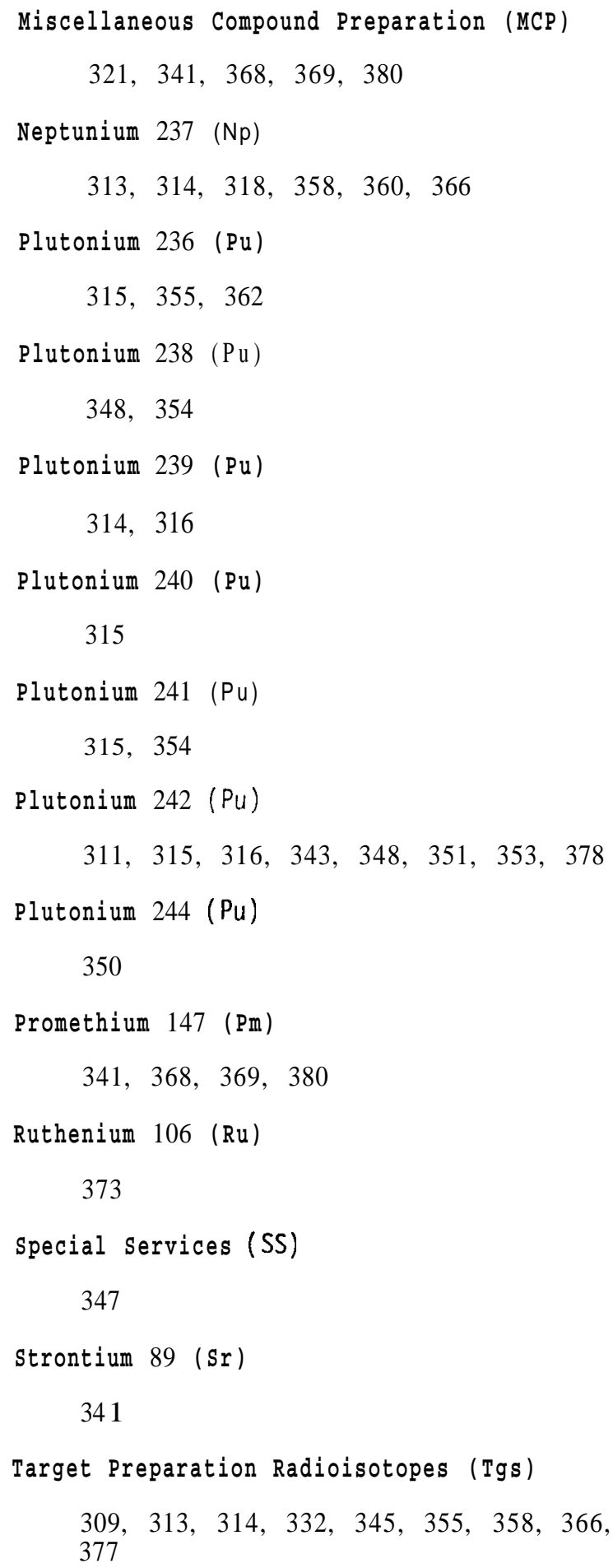


111. ALPHABETICAL LIST OF STATES AND FOREIGN COUNTRIES

CROSS-REFERENCED TO CUSTOMER NUMBERS

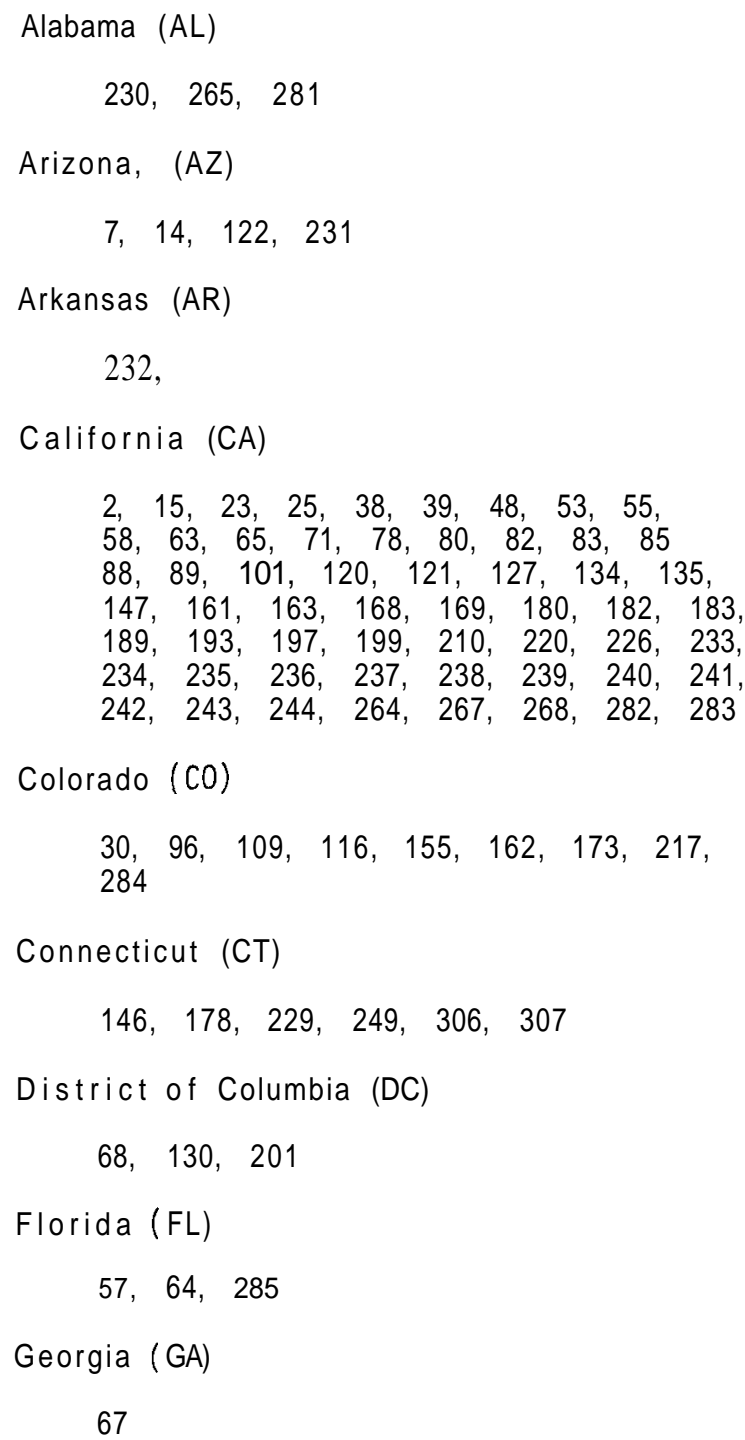

$8,9,11,33,44,61,113,125,144$,

$145,175,198,221,245,246,247,248$, 252, 286

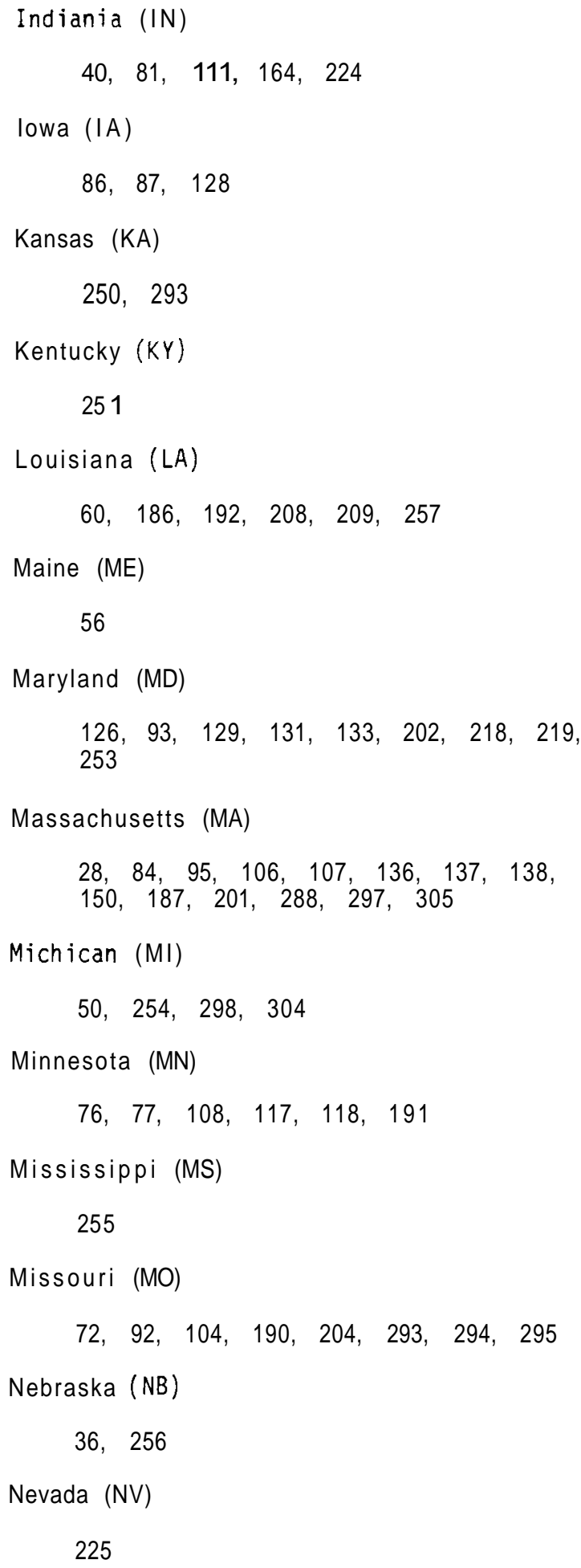




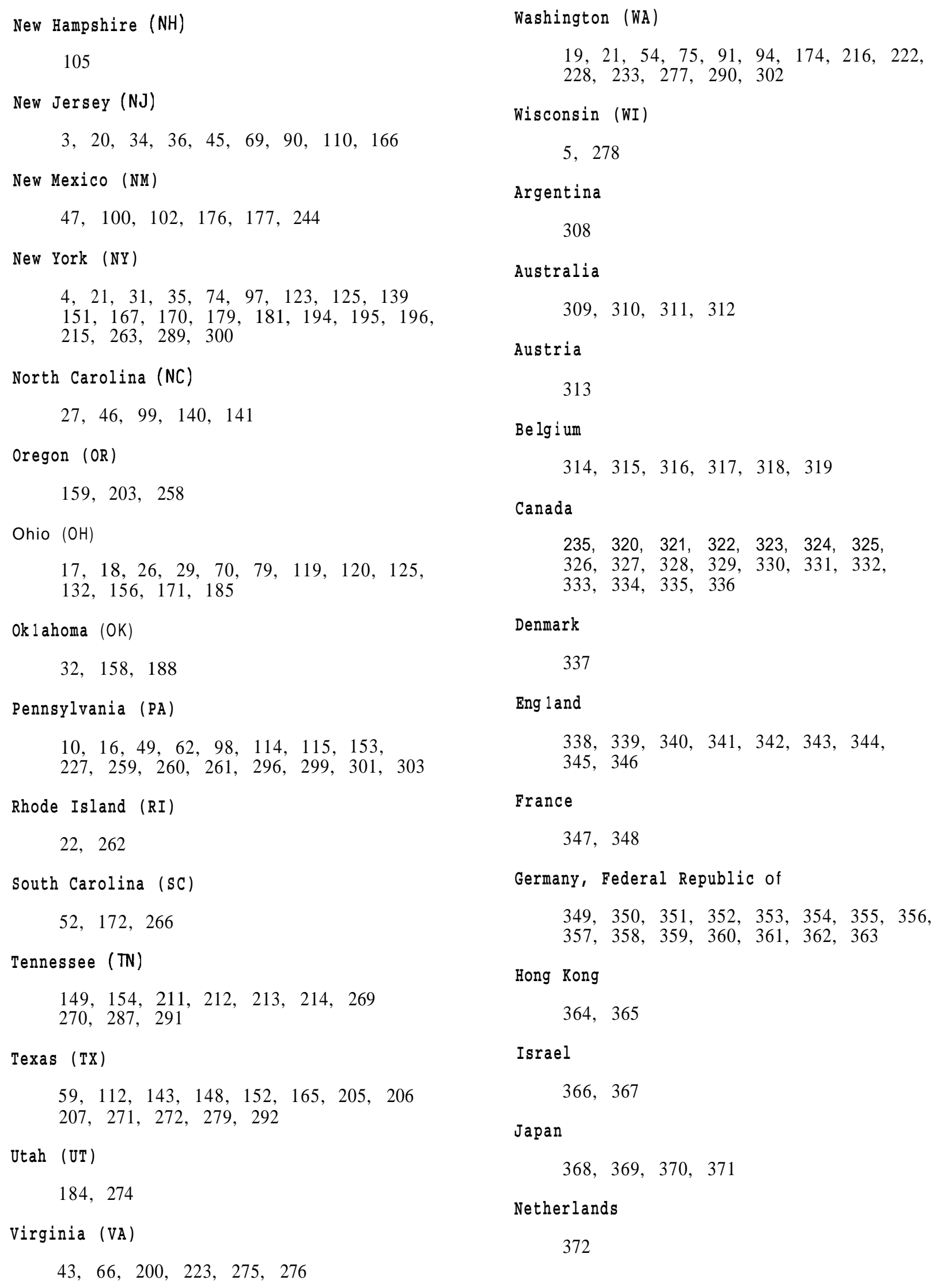

Washington (WA)

19, 21, 54, 75, 91, 94, 174, 216, 222, $228, \quad 233, \quad 277,290,302$

Wisconsin (WI)

5, 278

Argentina

308

Australia

$309,310,311,312$

Austria

313

Belg i um

$314,315,316,317,318,319$

Canada

$235,320,321,322,323,324,325$,

$326,327,328,329,330,331,332$,

$333,334,335,336$

Denmark

337

Eng 1 and

$338,339,340,341,342,343,344$, 345,346

France

347,348

Germany, Federal Republic of

$349,350,351,352,353,354,355,356$,

$357,358,359,360,361,362,363$

\section{Hong Kong}

364,365

Israel

366,367

Japan

$368, \quad 369, \quad 370, \quad 371$

Netherlands

372 
New Zealand

373

Norway

$374, \quad 375$

Scot 1and

376
Sweden

377

Switzerland

$378, \quad 379, \quad 380$

Taiwan

381 
IV. RADIOISOTOPE SALES AND TRANSFERS - FY 1978.

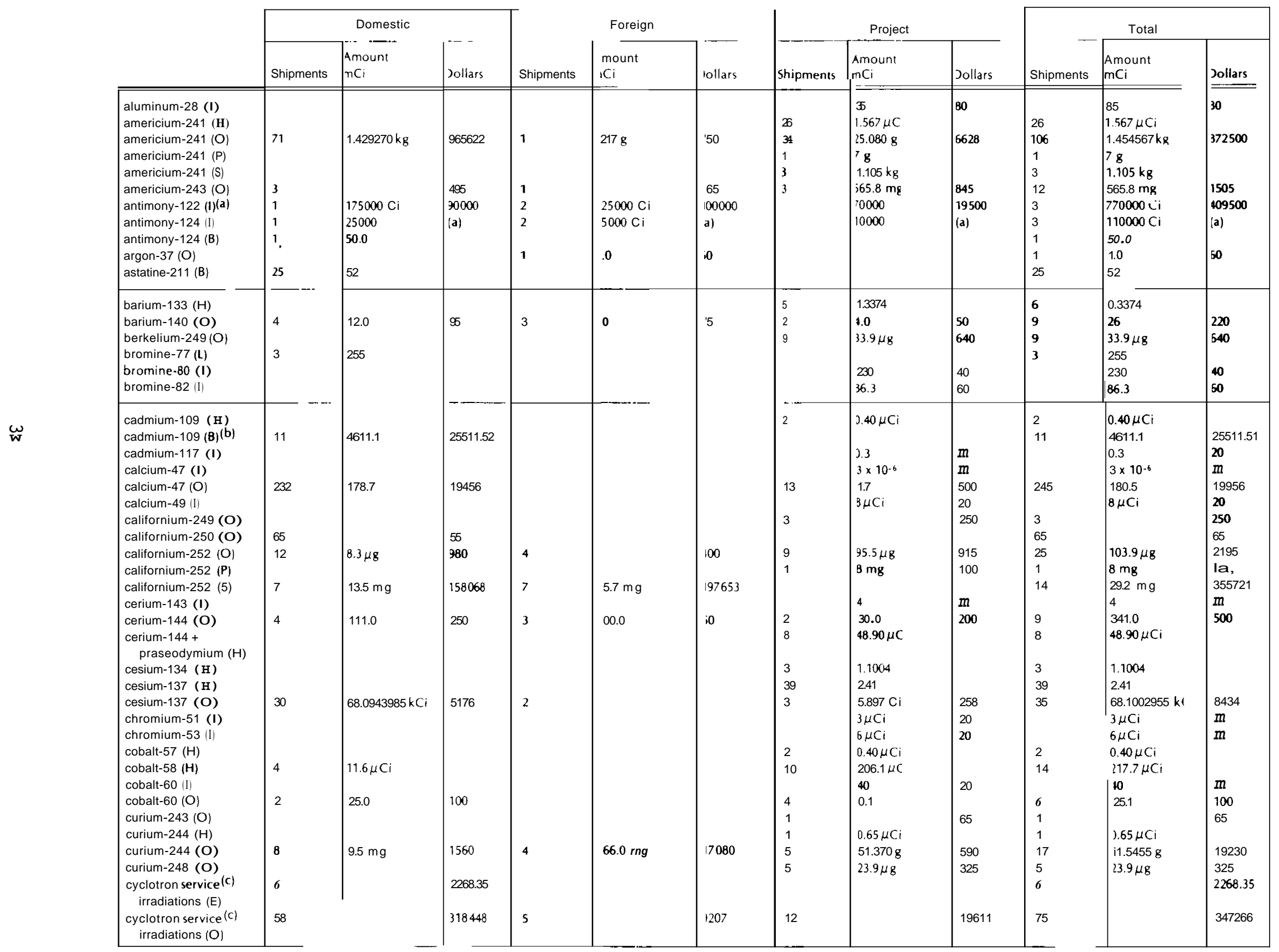


IV. RADIOISOTOPE SALES AND TRANSFERS - FY 1978. (continued)

\begin{tabular}{|c|c|c|c|c|c|c|c|c|c|c|c|c|}
\hline & \multicolumn{3}{|c|}{ Domestic } & \multicolumn{3}{|c|}{ Foreign } & \multicolumn{3}{|c|}{ Project } & \multicolumn{2}{|r|}{ Total } & \multirow{3}{*}{ Dollars } \\
\hline & Shipments & $\begin{array}{l}\text { Amount } \\
\mathrm{nCi}\end{array}$ & Oollars & Shipments & $\begin{array}{l}\text { Amount } \\
\mathrm{mCi}\end{array}$ & Dollars & : hipmenı & $\begin{array}{l}\text { Aınount } \\
\mathrm{mCi}\end{array}$ & Jollars & Shipments & $\begin{array}{l}\text { Amount } \\
\mathrm{mCi}\end{array}$ & \\
\hline depleted uranium $(P)$ & 19 & $1888 \mathrm{~kg}$ & 3774 & & & & 47 & $4249 \mathrm{~kg}$ & 1308 & 56 & $6137 \mathrm{~kg}$ & \\
\hline $\begin{array}{l}\text { einsteinium-253 }(\mathrm{O}) \\
\text { enriched uranium }(\mathrm{P}) \\
\text { encapsulation }(\mathrm{O}) \\
\text { europium-152 }(\mathrm{H}) \\
\text { europium-152(O) }\end{array}$ & 26 & $\begin{array}{l}125.307 \mathrm{~kg} \\
1500.0\end{array}$ & $\begin{array}{l}1065080 \\
31480 \\
30\end{array}$ & 3 & $10.261 \mathrm{~kg}$ & $\begin{array}{l}137180 \\
42400\end{array}$ & $\begin{array}{l}4 \\
33 \\
4 \\
1 \\
1\end{array}$ & $\begin{array}{l}0.4 \mathrm{mg} \\
70.740 \mathrm{~kg} \\
\\
0.1 \mu \mathrm{Ci} \\
10.0\end{array}$ & $\begin{array}{l}260 \\
601260 \\
22799 \\
200\end{array}$ & $\begin{array}{l}4 \\
62 \\
4 \\
1 \\
2\end{array}$ & $\begin{array}{l}0.4 \\
206.308 \mathrm{~kg} \\
\\
\mathbf{0 . 1} \boldsymbol{\mu \mathrm { Ci }} \\
1510.0\end{array}$ & $\begin{array}{l}175352 \\
146679\end{array}$ \\
\hline $\begin{array}{l}\text { fermium-257 (O) } \\
\text { fluorine-18 (8) }\end{array}$ & 8 & m7.5 & & & & & 2 & & 130 & $\begin{array}{l}2 \\
8\end{array}$ & 207.5 & 130 \\
\hline $\begin{array}{l}\text { gadolinium-153 (O) } \\
\text { germanium-68 (B) } \\
\text { gold-198 (I) }\end{array}$ & $\begin{array}{l}5 \\
2\end{array}$ & $\begin{array}{l}3601.0 \\
150.0\end{array}$ & 3850 & 5 & 21600 & 2160 & 2 & $\begin{array}{l}2002.0 \\
133\end{array}$ & $\begin{array}{l}2250 \\
40 \\
\end{array}$ & $\begin{array}{l}12 \\
2\end{array}$ & \begin{tabular}{|l|}
7763.0 \\
150.0 \\
133 \\
\end{tabular} & $\begin{array}{l}8260 \\
40 \\
\end{array}$ \\
\hline $\begin{array}{l}\text { lodine-123 }(\mathrm{E}) \\
\text { iodine-125 (8) } \\
\text { iodine-129 (O) } \\
\text { iodine-131 (O) } \\
\text { iridium-192(O) } \\
\text { iridium-192 meta }(\mathrm{O}) \\
\text { iron-52 (L) } \\
\text { iron-52 (B) } \\
\text { iron-55 (H) } \\
\text { iron-59 (I) }\end{array}$ & $\begin{array}{l}14 \\
1 \\
20 \\
6 \\
1 \\
160 \\
1 \\
3\end{array}$ & $\begin{array}{l}780.3 \\
2.0 \\
11.6855 \mathrm{~g} \\
754.0 \\
1.0 \\
722.241 \mathrm{kCi} \\
\mathbf{3 0} \\
88\end{array}$ & $\begin{array}{l}10042 \\
775 \\
25 \\
1069341\end{array}$ & 41 & $11.851 \mathrm{~g}$ & 1901 & $\begin{array}{l}1 \\
79\end{array}$ & $\begin{array}{l}201.0 \mathrm{mg} \\
1231.5 \\
\\
\\
\\
265.1 \\
0.1 \mu \mathrm{Ci} \\
\end{array}$ & $\begin{array}{l}210 \\
18915 \\
\\
\\
20 \\
\end{array}$ & \begin{tabular}{|l}
14 \\
1 \\
25 \\
85 \\
1 \\
160 \\
1 \\
3 \\
3
\end{tabular} & \begin{tabular}{|l}
780.3 \\
2.0 \\
$13.7465 \mathrm{~g}$ \\
1985.5 \\
1.0 \\
$722.241 \mathrm{kCi}$ \\
30 \\
$\mathbf{8 8}$ \\
265.1 \\
$0.1 \mu \mathrm{Ci}$ \\
\end{tabular} & \begin{tabular}{|l}
12153 \\
19690 \\
25 \\
106934 \\
\\
\\
$\mathrm{~m}$ \\
\end{tabular} \\
\hline $\begin{array}{l}\text { krypton-81 (B) } \\
\text { krypton-85 enriched }(\mathrm{O}) \\
\text { krypton-85 (O) }\end{array}$ & $\begin{array}{l}124 \\
1 \\
144\end{array}$ & $\begin{array}{l}3275.0 \\
25 \mathrm{Ci} \\
4.8647001 \mathrm{kCi}\end{array}$ & $\begin{array}{l}-1500 \\
107084\end{array}$ & 16 & $971 \mathrm{Ci}$ & 21362 & 42 & $5.1461825 \mathrm{Ci}$ & 56286 & $\begin{array}{l}124 \\
1 \\
202\end{array}$ & $\begin{array}{l}3275.0 \\
25 \mathrm{Ci} \\
10.9818826 \mathrm{kCi}\end{array}$ & $\begin{array}{l}1500 \\
194732\end{array}$ \\
\hline lanthanum-140 (I) & & & & & & & & 3.6 & 40 & & 3.6 & 40 \\
\hline $\begin{array}{l}\text { magnesium-28 (B) }(\mathbf{b}) \\
\text { manganese-54 }(\mathrm{H}) \\
\text { manganese-56 }(\mathrm{H}) \\
\text { manganese-56 (I) } \\
\text { miscellaneous corn- } \\
\text { pound preparations (O) } \\
\text { molybdenum-99 (I) }\end{array}$ & $\begin{array}{l}37 \\
2 \\
3 \\
30\end{array}$ & $\begin{array}{l}18.0 \\
3.3 \mu \mathrm{Ci} \\
8.3 \mu \mathrm{Ci} \\
\\
290.0\end{array}$ & $\begin{array}{l}6370 \\
1144\end{array}$ & 10 & 42 & 1813 & $\int_{10}^{15}$ & $\begin{array}{l}452.3 \mu \mathrm{Ci} \\
65.1 \mu \mathrm{Ci} \\
46.4 \\
10.0 \\
\\
2.4\end{array}$ & $\begin{array}{l}180 \\
2600 \\
120\end{array}$ & $\begin{array}{l}47 \\
17 \\
7 \\
40\end{array}$ & $\begin{array}{l}22.2 \\
455.5 \mu \mathrm{Ci} \\
73.4 \mu \mathrm{Ci} \\
46.4 \\
300.0 \\
2.4\end{array}$ & $\begin{array}{l}8183 \\
180 \\
3744 \\
120\end{array}$ \\
\hline $\begin{array}{l}\text { natural uranium }(\mathrm{H}) \\
\text { natural uranium }(\mathrm{P}) \\
\text { neodymium-147 (I) } \\
\text { neodymiun-149 (I) } \\
\text { neodymium-151 (I) }\end{array}$ & 4 & & & & & & 29 & $\begin{array}{l}18.5003 \mathrm{~g} \\
5 \\
1.5 \\
1.5\end{array}$ & $\begin{array}{l}40 \\
10 \\
40\end{array}$ & 7 & $\begin{array}{l}18.5003 \mathrm{~g} \\
5 \\
1.5 \\
1.5\end{array}$ & $\begin{array}{l}40 \\
40 \\
40\end{array}$ \\
\hline
\end{tabular}


IV. RADIOISOTOPE SALES AND TRANSFERS - FY 1978. (continued)

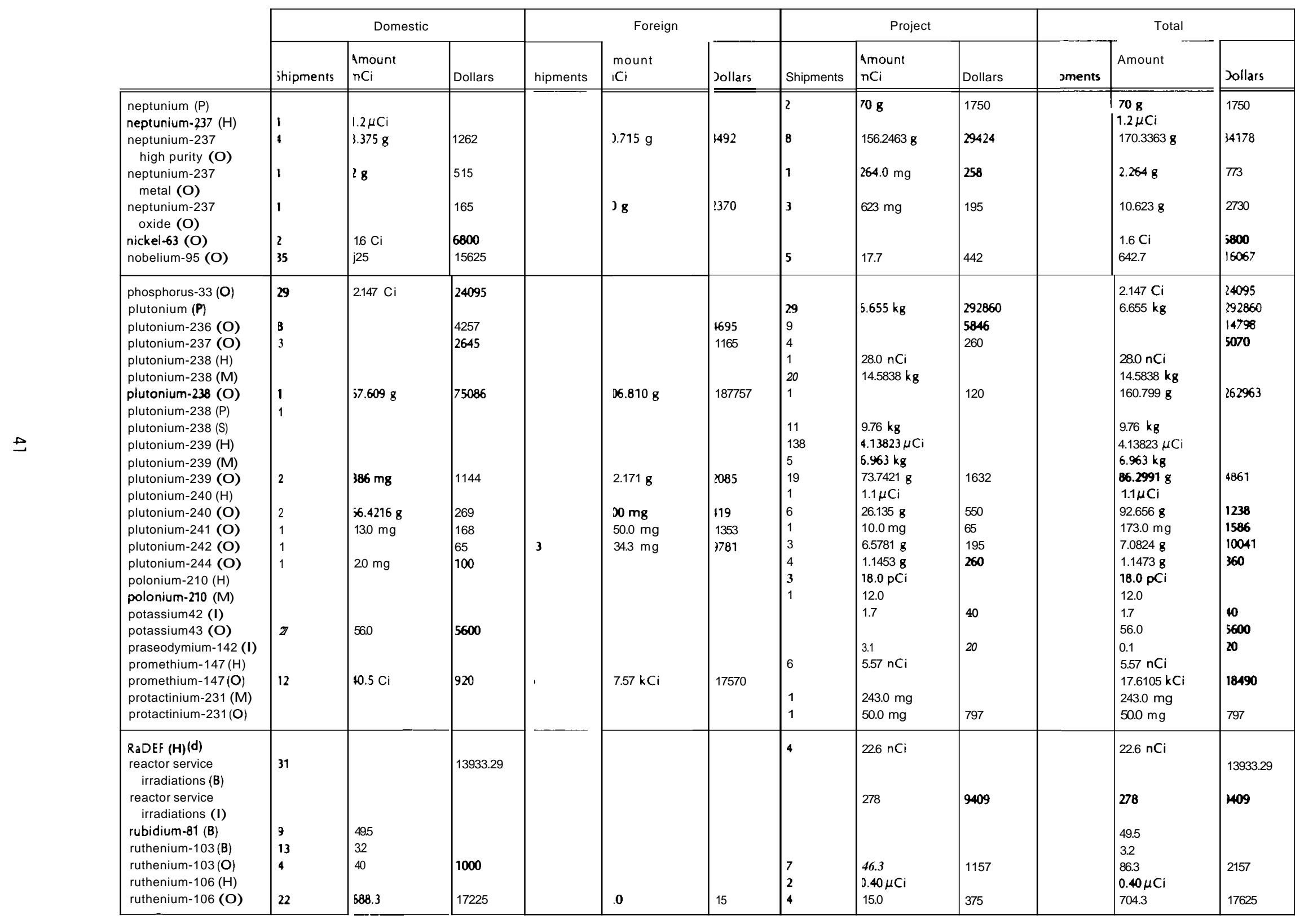


IV. RADIOISOTOPE SALES AND TRANSFERS - FY 1978. (continued)

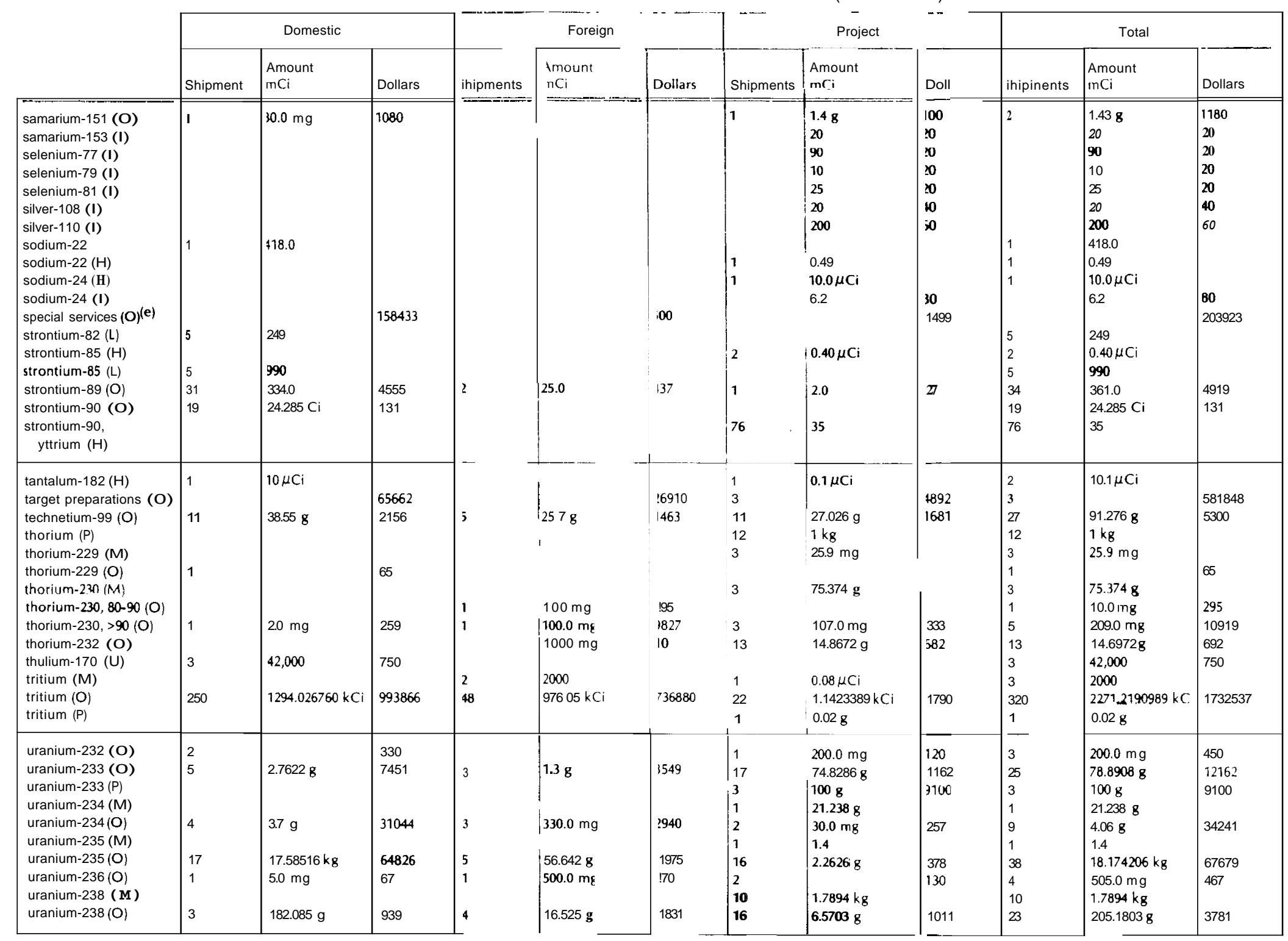


IV. RADIOISOTOPE SALES AND TRANSFERS - FY 1978. (continued)

\begin{tabular}{|c|c|c|c|c|c|c|c|c|c|c|c|c|}
\hline & \multicolumn{3}{|c|}{ Domestic } & \multicolumn{3}{|c|}{ Foreign } & \multicolumn{3}{|c|}{ Project } & \multicolumn{3}{|c|}{ Total } \\
\hline & Shipments & $\begin{array}{l}\text { Amount } \\
\mathrm{mCi}\end{array}$ & Dollars & Shipments & $\begin{array}{l}\text { Amount } \\
\mathrm{mCi}\end{array}$ & Dollars & Shipments & $\begin{array}{l}\text { Amount } \\
\mathrm{mCi}\end{array}$ & Dollars & Shipments & $\begin{array}{l}\text { Amount } \\
\mathrm{mCi}\end{array}$ & Dollars \\
\hline $\begin{array}{l}\text { withdrawn radio- } \\
\text { isotopes }\end{array}$ & 9 & $34.197930 \mathrm{kCi}$ & 50999 & & & & & & & 9 & $34.197930 \mathrm{kCi}$ & 50999 \\
\hline $\begin{array}{l}\text { xenon-127(B)(b) } \\
\text { xenon-128(l) } \\
\text { xenon-133(O) }\end{array}$ & $\begin{array}{l}200 \\
1000\end{array}$ & $\begin{array}{l}37560.4 \\
8.313201 \mathrm{~kg}\end{array}$ & $\begin{array}{l}60561 \\
545215\end{array}$ & 91 & $\begin{array}{l}11653.9 \\
94 \mathrm{~g}\end{array}$ & $\begin{array}{l}18837 \\
7625\end{array}$ & 18 & $\begin{array}{l}2216 \mathrm{cc} \\
13.120 \mathrm{~g}\end{array}$ & \begin{tabular}{|l|l}
151395 \\
1555
\end{tabular} & $\begin{array}{l}248 \\
1109\end{array}$ & $\begin{array}{l}49214.3 \\
2216 \mathrm{cc} \\
8.420321 \mathrm{~kg}\end{array}$ & $\begin{array}{l}79398 \\
151395 \\
554395\end{array}$ \\
\hline yutrium-91 (O) & 3 & 13.0 & 143 & 1 & 14.0 & 154 & 1 & 3.0 & 33 & 5 & 3.0 & 330 \\
\hline $\begin{array}{l}\text { zinc-65 }(\mathrm{H}) \\
\text { zirconium-95, } \\
\quad \text { niobium-95 (O) }\end{array}$ & 7 & 95.0 & 522 & 10 & 185.0 & 1017 & 8 & $\begin{array}{l}0.42 \mu \mathrm{Ci} \\
44.0\end{array}$ & 273 & $\begin{array}{l}2 \\
25\end{array}$ & $\begin{array}{l}0.42 \mu \mathrm{Ci} \\
324.0\end{array}$ & 1812 \\
\hline Totals & 2951 & & 6001518.16 & 338 & & 1727788 & 1045 & & 1825743 & 4334 & & 9555049.16 \\
\hline $\begin{aligned} A & =\text { Argonne Nation } \\
P & =\text { Battelle, Pacific } \\
B & =\text { Brookhaven Na } \\
H & =\text { Hanford Engine } \\
1 & =\text { Idaho Operatio }\end{aligned}$ & $\begin{array}{l}\text { oratory } \\
\text { west Laborat } \\
\text { Laboratory } \\
\text { Developmen } \\
\text { ice }\end{array}$ & $\begin{array}{l}\text { ories } \\
\text { Laboratory }\end{array}$ & & $\begin{aligned} M & =\text { Mou } \\
O & =\text { Oak } \\
S & =\text { Sava } \\
U & =U N C\end{aligned}$ & $\begin{array}{l}\text { nd Facility } \\
\text { Ridge Nati } \\
\text { nnah River } \\
\text { Nuclear I }\end{array}$ & boratory & & & & & & \\
\hline
\end{tabular}

\footnotetext{
(a) Dollar totals included with antimony-122 (1)

(b) Billed through Oak Ridige

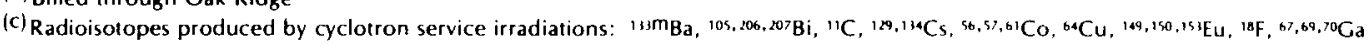

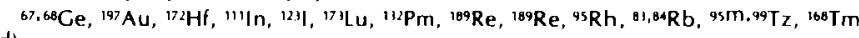

(d) $210 \mathrm{PO}_{\mathrm{O}} \rightarrow 210 \mathrm{Bi} \rightarrow 210 \mathrm{~Pb}$

(e) Special services include such items as target fabrication, source encapsulation, and preparation of special chemical forms. 
$\underline{P N L-2930}$

UC-23

\section{DISTRIBUTION}

No. of

Copies

OFFS ITE

J. C. Haugen

Argonne National Laboratory

9700 South Cass Avenue

Argonne, IL 60439

P. Colsmann

Brookhaven National Laboratory

Associated Universities, Inc.

Upton, NY 11973

P. Richards

Brookhaven National Laboratory

Associated Universities, Inc.

Upton, NY 11973

L. G. Stang, Jr.

Brookhaven National Laboratory

Associated Universities, Inc.

Upton, NY 11973

J. A. Morley

DOE Albuquerque Operations Office

PO Box 5400

Albuquerque, NM 87115

D. C. Graves

DOE Albuquerque Operations Office

PO Box 5400

Albuquerque, NM 87115

B. C. Blanke

DOE Dayton Area Office

PO Box 66

Miamisburg, $\mathrm{OH} 45342$

W. Ellgen

DOE Office of the Assistant Secretary for International Affairs (MS-7220C)

Washington, DC 20545

J. A. Garrett

DOE Office of the Assistant Secretary for International Affairs (MS-7220C)

Washington, DC 20545

W. A. Brobst

DOE Division of Environmental

Contro 1 Technology (MS-E201)

Washington, DC 20545

D. E. Large

DOE Oak Ridge Operations Office

PO Box E

Oak Ridge, TN 37830
No. of

Copies

OFFSITE

J. W. Nehls

DOE Oak Ridge Operations Office

PO Box E

Oak Ridge, TN 37830

10 J. N. Maddox

DOE Office of Program Coordination (MS-E201)

Office of the Assistant Secretary for Environment

Washington, DC 20545

P. B. Brimhall

DOE Idaho Operations Office

550 Second Street

Idaho Falls, ID 83401

W. K. Benson

DOE Division of International

Security Affairs (MS-E171)

Washington, DC 20545

J. E. Reid

DOE Office of Controller (MS-C217)

Washington, DC 20545

R. H., Schoonmaker

DOE Office of Controller (MS-D212)

Washington, DC 20545

E. L. Sillcox

DOE/OBP

Forrestal Bldg. (MS-8F031)

Washington, DC 20545

W. D. Sandberg

DOE Savannah River Operations Office

PO Box A

Aiken, SC 29801

27 DOE Technical Information Center

PO Box 62

Oak Ridge, $\mathbb{T N} 37830$

R. A. Hasse

Idaho National Engineering Company

EG\&G Idaho Inc.

PO Box 1625

Idaho Falls, ID 83401

E. W. Yeats

Lawrence Berkeley Laboratory

University of California

Berkeley, CA 94720 
No. of

Copies

OFFSITE

2 J. W. Meadows

Lawrence Livermore Laboratory

University of California

PO Box 808

Livermore, CA 94550

N. A. Matwiyoff

Los Alamos Scientific Laboratory

University of California

PO Box 1663

Los Alamos, NM 87544

H. A. O'Brien

Los Alamos Scientific Laboratory

University of California

PO Box 1663

Los Alamos, NM 87544

W. T. Cave

Mound Facility

Monsanto Research Corporation

P0 Box 32

Miamisburg, $\mathrm{OH} 45342$

R. DeWitt

Mound Facility

Monsanto Research Corporation

PO Box 32

Miamisburg, $\mathrm{OH} 45342$

R. E. Vallee

Mound Faci1ity

Monsanto Researcn Corporation

P0 Box 32

Miamisburg, $\mathrm{OH} 45342$

H. R. Gwinn

Oak Ridge National Laboratory

Union Carbide Corporation

PO Box X

Oak Ridge, TN 37830

E. H. Kobisk

Oak Ridge National Laboratory

Union Carbide Corporation

PO Box X

Oak Ridge, TN 37830

E. E. Lamb

Oak Ridge National Laboratory

Union Carbide Corporation

PO Box $X$

Oak Ridge, TN 37830

E. Newman

Oak Ridge National Laboratory

Union Carbide Corporation

PO Box X

Oak Ridge, Tn 37830
No. of

$\underline{\text { Copies }}$

$\underline{\text { OFFSI TE }}$

5 J. E. Ratledge

Oak Ridge National Laboratory

Union Carbide Corporation

PO Box X

Oak Ridge, $\mathbb{T N} 37830$

E. Vejvoda

Rockwell International

Atomics International Division

Rocky Flats Plant

PO Box 464

Golden, $\quad$ CO 80401

K. W. MacMurdo

Savannah River Laboratory

E. I. du Pont de Nemours and Company

Aiken, SC 29801

0. H. Turno

Savannah River Laboratory

E. I. du Pont de Nemours and Company

Aiken, SC 29801

A. F. Grella

U.S. Department of Transportation

Washington, DC 20555

R. F. Barker

U.S. Nuclear Regulatory Commission

Washington, DC 20555

D. R. Hopkins

U.S. Nuclear Regulatory Commission

Washington, OC 20555

E. P. Resner

U.S. Nuclear Regulatory Commission

Washington, DC 20555

B. Singer

U.S. Nuclear Regulatory Commission Washington, DC 20555

A. N. Tse

U.S. Nuclear Regulatory Commission Washington, DC 20555

E. D. Flack

U.S. Nuclear Reaulatory Commission Office of Inspection and Enforcement E/W 359

Washington, DC 20555

R. E. Devine

U.S. Nuclear Regulatory Commission

Library

Washington D.C. 20555 
No. of

$\underline{\text { Copies }}$

OFFS ITE

G. Smith

U.S. Nuclear Regulatory Commission

631 Park Avenue

King of Prussia, PA 19406

J. Suther 1and

U.S. Nuclear Regulatory Commission

101 Marietta Street

Suite 3100

Atlanta, GA 30303

B. Davis

U.S. Nuclear Regulatory Commission

799 Roosevelt Road

Glen Ellyn, IL 60137

Donald Hamilton

1901 Chapman Avenue

Mailstop HFX-70

Rockville, MD 20857

G. Brown

U.S. Nuclear Regulatory Commission 611 Ryan Plaza Drive

Suite 1000

Arlington, TX 76012

\section{H. Book}

U.S. Nuclear Regulatory Commission 1990 N. California Blvd.

Suite 202

Walnut Creek, CA 94596
No. of

Copies

\section{ONSITE}

2 DOE Richland Operations Office

W. R. Pohlod

H. E. Ransom

1 Hanford Engineering Development Laboratory

D. Engel

1 UNC Nuclear Industries

P. A. Carlson

34 Pacific Northwest Laboratory

W. J. Bair

J. S. Burlison (20)

J. J. Fuquay

J. H. Jarrett

R. W. McKee

R. W. Perk ins

D. B. Shipler

E. J. Wheelwright

Publishing Coordination (2)

Technical Information (5) 